\title{
Isogeometric approximation of cardiac electrophysiology models on surfaces: an accuracy study with application to the human left atrium
}

\author{
Alessandro S. Patelli ${ }^{\mathrm{a}}$, Luca Dedè ${ }^{\mathrm{b}, *}$, Toni Lassila ${ }^{\mathrm{c}}$, Andrea Bartezzaghi $^{\mathrm{b}}$, Alfio Quarteroni ${ }^{\mathrm{b}, \mathrm{d}}$ \\ ${ }^{a}$ Laboratory for Computation and Visualization in Mathematics and Mechanics, \\ École Fédérale Polytechnique de Lausanne, Station 8, EPFL, CH-1015 Lausanne, Switzerland \\ ${ }^{b}$ Chair of Modeling and Scientific Computing, Mathematics Institute of Computational Science and Engineering, \\ École Fédérale Polytechnique de Lausanne, Station 8, EPFL, CH-1015 Lausanne, Switzerland \\ ${ }^{c}$ Computational Imaging 85 Simulation Technologies in Biomedicine, Department of Electronic and Electrical Engineering, \\ University of Sheffield, Pam Liversidge Building, Mappin Street, Sheffield S1 3JD Sheffield, United Kingdom \\ ${ }^{d}$ MOX-Modeling and Scientific Computing, Mathematics Department "F. Brioschi", \\ Politecnico di Milano, via Bonardi 9, Milano, 20133, Italy (on leave)
}

\begin{abstract}
We consider Isogeometric Analysis in the framework of the Galerkin method for the spatial approximation of cardiac electrophysiology models defined on NURBS surfaces; specifically, we perform a numerical comparison between basis functions of degree $p \geq 1$ and globally $C^{k}$-continuous, with $k=0$ or $p-1$, to find the most accurate approximation of a propagating front with the minimal number of degrees of freedom. We show that B-spline basis functions of degree $p \geq 1$, which are $C^{p-1}$-continuous capture accurately the front velocity of the transmembrane potential even with moderately refined meshes; similarly, we show that, for accurate tracking of curved fronts, high-order continuous B-spline basis functions should be used. Finally, we apply Isogeometric Analysis to an idealized human left atrial geometry described by NURBS with physiologically sound fiber directions and anisotropic conductivity tensor to demonstrate that the numerical scheme retains its favorable approximation properties also in a more realistic setting.
\end{abstract}

Keywords: Isogeometric Analysis, cardiac electrophysiology, surface PDEs, high-order approximation

\section{Introduction}

The heart is a muscular organ that contracts due to a signal originating from the heart's natural pacemaker, the sinoatrial node, that enters the cardiac muscle through the His-Purkinje system. Once the electrical signal has entered the muscle, it travels on the cell membrane of the cardiac muscle cells (cardiomyocytes) and passes from cell to cell through the gap junctions. Under the so-called action potential the

\footnotetext{
${ }^{*}$ Corresponding author

Email addresses: alessandro.patelli@epfl.ch (Alessandro S. Patelli), luca.dede@epfl.ch (Luca Dedè), t.lassila@sheffield.ac.uk (Toni Lassila), andrea.bartezzaghi@epfl.ch (Andrea Bartezzaghi), alfio.quarteroni@epfl.ch (Alfio Quarteroni)
} 
individual cells rapidly become depolarized as positively charged ions enter the cell, triggering the contraction of the cellular contractile units called sarcomeres. After a period of contraction the positively charged ions are pumped out of the cells and they repolarize to their resting potential, allowing the muscle to relax and wait for the next signal to contract again after a refractory period during which no excitation can take place. For insight on the physiological processes of cardiac activation, we refer to [35].

Mathematical modelling of electrophysiology has shown great promise in being a viable diagnosis and prediction tool that in the future may be used to guide clinical decision making $[20,53,55,64]$. The standard mathematical model for cardiac electrophysiology is the bidomain model, where the tissue is conceptually divided into the intracellular and extracellular spaces. A formal homogenization procedure is followed to reduce away the microstructure of the cells and leads to a system of two reaction-diffusion equations for the intracellular and extracellular potentials. A further assumption of equal anisotropic conductivities in the intra- and extracellular compartments leads to a simplified formulation in terms of the transmembrane potential that is defined as the difference of the two potentials, called the monodomain equation, requiring only the solution of a single reaction-diffusion equation. For details on the derivation of the equations and further bibliography on their mathematical approximation, see [19, 20].

The bi-/monodomain equation(s) need to be completed by a model describing the ionic currents passing through voltage-sensitive protein structures called ion channels that give rise to the action potential. Since any single cell has hundreds of channels that regulate the passage of numerous different molecular species through the cell membrane, a large number of different membrane models of varying complexity have been developed in order to describe the cellular excitation process at increasing levels of complexity. The end result is a (typically very stiff) system of ordinary differential equations that needs to be coupled to the bi-/monodomain equation(s). The derivation and analysis of the membrane models we consider in this paper can be found in [42].

At the mesoscopic level, cardiac tissue has a highly anisotropic structure. The cardiomyocytes are organized into laminar sheetlets, where the muscle cells are tubular in shape and roughly oriented in the same direction locally, called the (mean) fiber direction. Embedding the muscle cells is a gelatinous interstitial foam supported by a fibrous extracellular matrix formed mainly of collagen proteins that is synthesized by cardiac fibroblast cells. In the two atria (antechambers of the heart) the walls are considerably thinner than in the ventricles, yet exhibit similar anisotropic structure that is less well documented and understood due to its more complex nature [65]. In many cases the atrial walls are assumed to be thin enough such that a typical simplification is to consider them as surfaces (two-dimensional manifolds) and to formulate the bi-/monodomain equation(s) as surface PDEs. This is the approach taken in this work. For a recent review on the challenges of computational modelling of the atria necessary to capture other physiological aspects that are not treated in this work, we refer to [22]. 


\subsection{Challenges in numerical approximation of electrophysiology}

While the numerical discretization of the bi-/monodomain equation(s) and the related membrane model is straightforward, several numerical difficulties are known to exist. The solutions of these equations exhibit travelling pulse solutions with sharp wavefronts, especially for the more realistic stiff membrane models found in literature. Unless sufficiently accurate resolution of the travelling front is performed, inaccurate propagation velocity and/or dynamics are obtained and as a result incorrect predictions about the cardiac activation pattern are made. Since the front propagation velocity depends on its curvature, in the surface PDE formulation it is especially important to use a sufficiently smooth function space for the spatial approximation that minimizes the effect of the numerical grid.

Numerical approximation of the bi-/monodomain equation(s) still relies heavily on low-order spatial approximations combined with highly refined uniform meshes in order to capture correctly the front propagation. In full-heart human electrophysiology simulations such "overkill" meshes lead to systems of hundreds of millions of degrees of freedom. Approaches to improving the front approximation without excessive global refinement that have been suggested in the literature include modifying the quadrature rule for the ionic currents [43], applying mesh adaptivity near the front [9, 18, 61], and more recently using high-order Spectral Element discretizations [12]. In this work, we investigate an approach similar to the latter, except that we replace orthogonal polynomials with B-splines or Non-Uniform Rational B-splines (NURBS) [52] in the context of Isogeometric Analysis (IGA) in the framework of the Galerkin method [23, 38].

\subsection{Numerical approximation of electrophysiology using IGA}

IGA has been nowadays successfully used in a broad range of applications in virtue of its versatility and geometrical advantages. Indeed, IGA is based on the Isogeometric concept, a reversal of the Isoparametric paradigm, which facilitates the encapsulation of the exact geometrical representation into the approximation of the PDEs; specifically, in the Isogeometric concept the basis functions that are used for the geometrical representation of the computational domain of the PDEs are then also used for the approximation of their unknown solution fields. Since a broad range of geometries of practical interest are represented exactly by B-splines or NURBS, IGA commonly employs these as basis functions both in Galerkin $[8,38]$ and collocation [5, 59] methods. NURBS basis functions are built from B-splines, piecewise polynomials of degree $p$ and global continuity $C^{k}$, with $k$ ranging from 0 to $p-1$. The particularity of choosing the global regularity $k$ of the basis functions together with the degree $p$ is related to the $k$-refinement procedure, a combination of the $p$ - and $h$-refinement procedures exclusive of NURBS and often used in IGA [23] to obtain fine meshes with high order continuous basis functions of degree $p$. In this respect, the advantages of solving PDEs with regular solutions by means of high order continuous basis functions over their $C^{0}$ continuous counterparts have been extensively studied in the IGA community, especially in the framework of the Galerkin method; see e.g. [2, 24, 27, 30, 39, 40]. 
In this work, we consider the spatial approximation of the PDEs arising in electrophysiology models, specifically for the monodomain equation, by means of NURBS-based IGA in the framework of the Galerkin method with particular emphasis on the role of using NURBS basis functions of degree $p$ and high order global continuity $k=p-1$. With this aim, we perform a numerical study of the front propagation velocity when considering B-spline basis functions of different degrees and global continuity, especially highlighting the advantages of the $k$-refinement procedure typical of IGA; specifically, we consider both the MitchellSchaeffer [46] and Aliev-Panfilov models [3]. In addition, for the latter electrophysiology model, we focus on the role of high order continuous B-splines basis functions in the spatial representation of spiral waves, with particular emphasis on the accuracy and number of degrees of freedom associated to the discrete problem. We remark that, to the best of our knowledge, this is the first accuracy study for IGA in the context of cardiac electrophysiology; indeed, existing works [14, 15] rather focus on the definition of efficient and scalable preconditioners for the bidomain model [51, 60].

Throughout this work, we assume that the atria can be considered as thin-walled and thus are modeled as two-dimensional surfaces with anisotropy present only in the tangent plane. This assumption at first sight ignores the inherent transmural anisotropy that is present in the fiber structure of the atrial walls, but recent works $[13,21]$ have shown that a proper homogenization treatment of the transmural anisotropy allows the definition of an effective conductivity tensor that recovers the mid-wall activation pattern with sufficient accuracy. In this respect, IGA provides a natural framework for the spatial approximation of elecrophysiology models defined on surfaces, specifically when these are defined by NURBS, as highlighted in [6] and [28]. While sophisticated ionic models for the electrophysiology of the atria exist - e.g. the Nygren-Fiset-Firek-Clark-Lindblad-Clark-Giles model with 29 variables [48] or the Courtemanche-RamirezNattel model with 21 variables [26] — in this paper, we limit ourselves to consider the monodomain problem with the Mitchell-Schaeffer model in order to better highlight the approximation properties of IGA. We expect however that the benefits of the IGA approximation straightforwardly extend to more physically meaningful models for atrial electrophysiology [32].

This paper is organized as follows. In Sect. 2 we provide the mathematical formulation of the monodomain problem as surface PDEs, some examples of membrane models that can be used to simulate atrial activation, namely the Mitchell-Schaeffer and Aliev-Panfilov models, and briefly recall their spatial discretization by using NURBS-based IGA. In Sect. 3 we perform a convergence study of the discrete conduction velocity in order to demonstrate the improved accuracy of propagation velocity using IGA with high order continuous NURBS basis functions. We also demonstrate certain low order continuous spatial discretizations may exhibit grid imprinting in the sense that complex spiral wave dynamics tends to incorrectly follow the numerical gridlines, whereas sufficiently high order approximations correctly approximate the periodic dynamics without the need to significantly increase the size of the discrete problem. In Sect. 4 a construction of an idealized human left atrium geometry using NURBS is performed to consider a realistic test case. An 
indicative fiber geometry is prescribed on the atrial surface and used to perform tests on the effect of spatial accuracy for NURBS-based IGA. Conclusions follow in Sect. 5.

\section{Numerical approximation of the cardiac electrophysiology problem}

\subsection{Single-cell electrophysiology models}

Models for the cardiac excitation at the cellular level are typically based on the Hodgkin-Huxley formalism of ionic currents passing through the cell membrane. For a model that contains $K$ different ion channels for different ionic species (the most important ones being $\mathrm{K}^{+}, \mathrm{Na}^{+}$, and $\mathrm{Ca}^{2+}$ ), a system of ordinary differential equations expressing the conservation of charges reads [19]:

$$
\left\{\begin{aligned}
C_{m} \frac{d v}{d t}+i_{\text {ion }}(v, \mathbf{w}, \mathbf{c}) & =i_{\text {app }}(t), \\
i_{\text {ion }}(v, \mathbf{w}, \mathbf{c}) & =\sum_{k=1}^{K} g_{k}(\mathbf{c}) \prod_{j=1}^{J} w_{j}^{p_{j_{k}}}\left(v-v_{k}(\mathbf{c})\right)+I_{0}(v, \mathbf{c}), \\
\frac{d w}{d t} & =m_{\mathbf{w}}(v, \mathbf{w}, \mathbf{c}), \\
\frac{d c}{d t} & =m_{\mathbf{c}}(v, \mathbf{w}, \mathbf{c}) .
\end{aligned}\right.
$$

The unknowns of the problem are $v$ the transmembrane potential between the intra- and extracellular spaces, $w_{j}$ the $J$ so-called recovery variables taking values in $[0,1]$ that regulate the transmembrane currents, and $c_{k}$ the $K$ intracellular concentrations of the different ionic species. The model parameters are $C_{m}$ the local membrane capacitance, $i_{\text {app }}$ the applied external current, and $v_{k}$ the reversal potential of the $k$ th ionic species. In addition, evolution equations need to be prescribed for the recovery variables and ionic concentrations, as symbolically indicated in the latter two relations of Eq. (1).

In this work, we use the simpler phenomenological models of Mitchell-Schaeffer [46] and Aliev-Panfilov [3], which only have two currents, inward and outward, one recovery variable $w$ and no explicit ionic concentration variables. The general form of these simplified single-cell models reads:

$$
\left\{\begin{array}{rlrl}
C_{m} \frac{d v}{d t}+i_{\text {ion }}(v, w) & =i_{\text {app }}(t) & & \text { in }(0, T), \\
\frac{d w}{d t} & =g_{\mathrm{rec}}(v, w) & & \text { in }(0, T), \\
v(0) & =v_{0}, & \\
w(0) & =w_{0}, &
\end{array}\right.
$$

where $v_{0}$ and $w_{0}$ are the initial values of the transmembrane potential and recovery variables, respectively. For the Mitchell-Schaeffer model we have:

$$
i_{\mathrm{ion}}(v, w):=\frac{v}{\tau_{\text {out }}}+\frac{w}{\tau_{\text {in }}} v^{2}(v-1) \quad \text { and } \quad g_{\mathrm{rec}}(v, w):=\left\{\begin{array}{cc}
\frac{1-w}{\tau_{\text {open }}} & \text { if } v<v_{\mathrm{rec}}, \\
\frac{-w}{\tau_{\text {close }}} & \text { if } v>v_{\mathrm{rec}},
\end{array}\right.
$$


while for the (linearized) Aliev-Panfilov model:

$$
i_{\text {ion }}(v, w):=k v(v-a)(1-v)-v w \quad \text { and } \quad g_{\text {rec }}(v, w):=\left(\epsilon_{0}+\frac{\mu_{1} w}{\mu_{2}+v}\right)(-w-k v(v-b-1)) .
$$

The parameters $\tau_{\text {out }}, \tau_{\text {in }}, \tau_{\text {open }}, \tau_{\text {close }}$, and $v_{\text {rec }}$ define the Mitchell-Schaeffer model, while $k, a, b, \epsilon_{0}, \mu_{1}$, and $\mu_{2}$ the Aliev-Panfilov model.

\subsection{Monodomain equation for tissue-level activation}

Starting from the single-cell model (2), we consider the monodomain problem for the tissue-level activation defined in the computational domain $\Omega \subset \mathbb{R}^{d}$, with $d=2$ or 3 . In the case $d=3$, we consider that $\Omega$ is a smooth submanifold of codimension one with regular boundary (an open surface), while for the case $d=2$ we consider that $\Omega$ is of codimension zero and is a bounded subdomain in the plane with regular boundary. Let us introduce the function spaces $L^{2}(\Omega)$ of square integrable functions in $\Omega, L^{\infty}(\Omega)$, and the Hilbert space $H^{1}(\Omega)$; see [1]. Then, the general form of the monodomain problem with the Mitchell-Schaeffer or Aliev-Panfilov model(s) reads:

$$
\text { find } v, w: \Omega \times(0, T) \rightarrow \mathbb{R}::\left\{\begin{aligned}
C_{m} \frac{\partial v}{\partial t} & =\nabla_{\Omega} \cdot\left(\mathbf{D} \nabla_{\Omega} v\right)-i_{\text {ion }}(v, w)+i_{\text {app }}(t) & & \text { in } \Omega \times(0, T), \\
& \text { boundary conditions } & & \text { on } \partial \Omega \times(0, T), \\
\frac{d w}{d t} & =g_{\mathrm{rec}}(v, w) & & \text { in } \Omega \times(0, T), \\
v(0) & =v_{0} & & \text { in } \Omega, \\
w(0) & =w_{0} & & \text { in } \Omega,
\end{aligned}\right.
$$

which is a system coupling a PDE for the transmembrane potential $v$ and an ODE for the recovery variable $w$. The second order tensor function $\mathbf{D} \in L^{\infty}\left(\bar{\Omega} ; \mathbb{R}^{d \times d}\right)$ is symmetric, uniformly elliptic, and represents the anisotropic conductivity whose expression will be specified later. The differential operator $\nabla_{\Omega}$ indicates the surface gradient operator, i.e. $\nabla_{\Omega} \phi: \Omega \rightarrow \mathbb{R}^{d}$ for $\phi \in C^{1}(\Omega)$; similarly, $\nabla_{\Omega}$. stands for the surface divergence operator, i.e. $\nabla_{\Omega} \cdot \mathbf{z}: \Omega \rightarrow \mathbb{R}$ for $\mathbf{z} \in\left[C^{1}(\Omega)\right]^{d}$ (see e.g. [10, 28, 29]). Suitable boundary conditions (essential or natural) must be specified on the boundary $\partial \Omega$ of $\Omega$, provided that the surface is not closed $(\partial \Omega \neq \emptyset)$.

We consider the weak formulation of the PDE in the monodomain problem (5) and define the trial space for the transmembrane potential $\mathcal{V} \subset C^{0}\left([0, T] ; L^{2}(\Omega)\right) \cap L^{2}\left((0, T) ; H^{1}(\Omega)\right)$ and the test space $\mathcal{V}_{0} \subseteq H_{0}^{1}(\Omega)$, which enforces the essential boundary conditions, the latter in homogeneous form; moreover, we assume the recovery variable $w \in \mathcal{W}$, with $\mathcal{W}$ a suitable Bochner function space, e.g. such that $\mathcal{W} \subseteq C^{0}\left([0, T] ; L^{\infty}(\Omega)\right)$ is sufficient to guarantee existence of weak solutions to the Mitchell-Schaeffer model [44], as well as to the Aliev-Panfilov model. Then, the nonlinear PDE appearing in problem (5) reads in weak formulation:

$$
\begin{aligned}
& \forall t \in(0, T) \text {, find } v(t) \in \mathcal{V} \quad: \\
& \qquad\left(\phi, C_{m} \frac{d v}{d t}(t)\right)+\left(\nabla_{\Omega} \phi, \mathbf{D} \nabla_{\Omega} v(t)\right)+\left(\phi, i_{\text {ion }}(v(t), \widetilde{w}(t))\right)-\left(\phi, i_{\text {app }}(t)\right)=0 \quad \forall \phi \in \mathcal{V}_{0},
\end{aligned}
$$




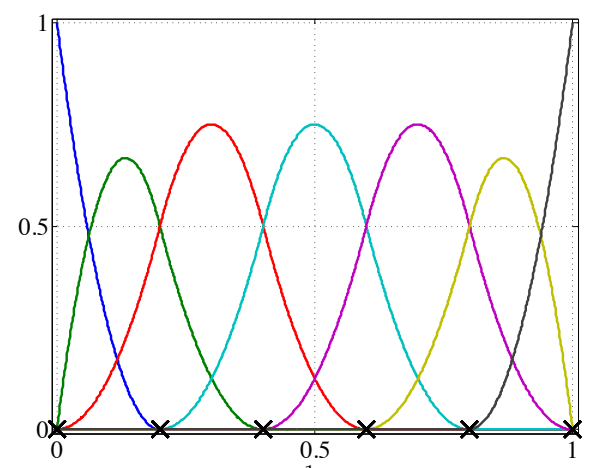

(a) $p=2, C^{1}$-continuous

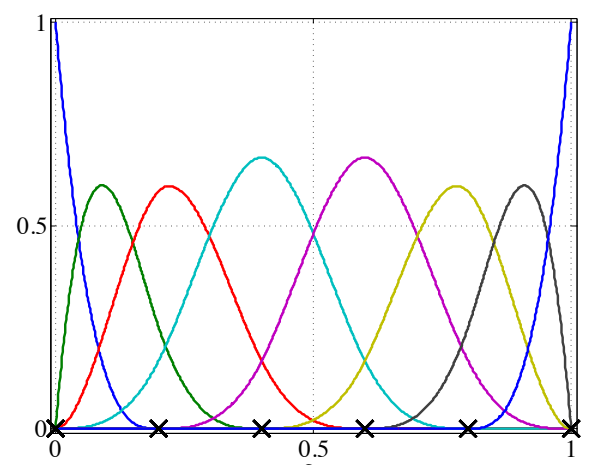

(c) $p=3, C^{2}$-continuous

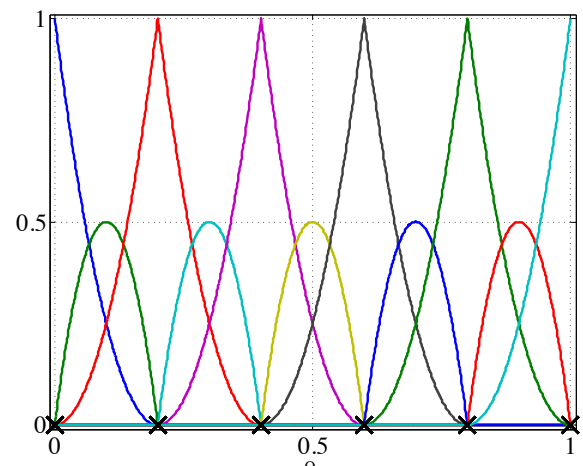

(b) $p=2, C^{0}$-continuous

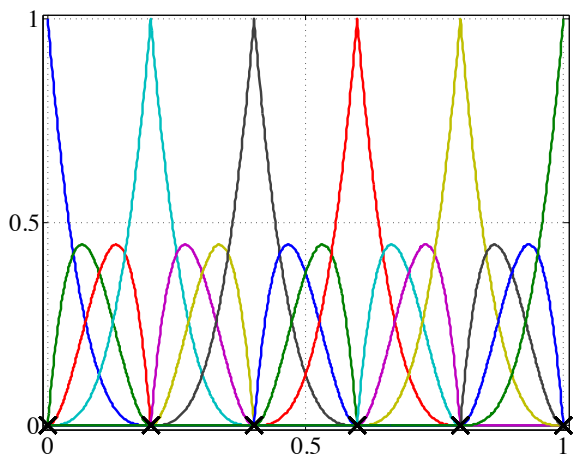

(d) $p=3, C^{0}$-continuous

Figure 1: Examples of univariate B-spline basis functions $\left\{\widehat{N}_{i}(\boldsymbol{\xi})\right\}_{i=1}^{n_{b f}}$ in $\widehat{\Omega}=(0,1)$ obtained from the knot vectors $\left\{\{0\}^{p+1},\left\{\frac{1}{5}\right\}^{p},\left\{\frac{2}{5}\right\}^{p},\left\{\frac{3}{5}\right\}^{p},\left\{\frac{4}{5}\right\}^{p}\{1\}^{p+1}\right\}$ for the degrees $p=2$ (top) and $p=3$ (bottom) and global $C^{\alpha}$-continuity with $\alpha=p-1$ (left) and $\alpha=0$ (right); the number of non-zero elements is $\widetilde{n}_{e l}=5$ and the number of basis functions are $n_{b f}=7,11,8$, and 16 from (a) to (d), respectively.

with $v(0)=v_{0}$ in $\Omega$, for any given function $\widetilde{w}(t) \in \mathcal{W}$, where $(\cdot, \cdot)$ indicates the standard $L^{2}(\Omega)$ scalar product. Finally, the full coupled monodomain problem (5) reads:

$$
\begin{aligned}
& \forall t \in(0, T) \text {, find } v(t) \in \mathcal{V}, w(t) \in \mathcal{W} \quad: \\
& \qquad \begin{aligned}
\left(\phi, C_{m} \frac{d v}{d t}(t)\right)+\left(\nabla_{\Omega} \phi, \mathbf{D} \nabla_{\Omega} v(t)\right) & \\
+\left(\phi, i_{\text {ion }}(v(t), w(t))\right)-\left(\phi, i_{\text {app }}(t)\right)=0 & \forall \phi \in \mathcal{V}_{0}, \\
\frac{d w}{d t}(t)-g_{\text {rec }}(v(t), w(t))=0 & \text { in } \Omega,
\end{aligned}
\end{aligned}
$$

with $v(0)=v_{0}$ and $w(0)=w_{0}$ in $\Omega$. We assume that the applied current $i_{\text {app }} \in L^{2}\left(0, T ; H^{-1}(\Omega)\right)$, the initial transmembrane potential $v_{0} \in L^{2}(\Omega)$, and the initial recovery variable $w_{0} \in L^{\infty}(\Omega)$, with $0 \leq w_{0} \leq 1$ to guarantee existence of weak solutions $[11,44]$ for all time.

\subsection{Spatial approximation: IGA for surface PDEs}

Let us assume that the computational domain $\Omega$ is a surface in $\mathbb{R}^{3}$ and that is geometrically represented 
by means of B-splines or, more generally, NURBS ([52]). In particular, the surface $\Omega \subset \mathbb{R}^{3}$ is defined in terms of the geometrical mapping $\mathbf{x}: \widehat{\Omega} \rightarrow \mathbb{R}^{3}, \boldsymbol{\xi} \rightarrow \mathbf{x}(\boldsymbol{\xi})$, where $\widehat{\Omega} \subset \mathbb{R}^{2}$ is a parametric domain and $\boldsymbol{\xi}=\left(\xi_{1}, \xi_{2}\right)^{T}$ a vector-valued independent variable; in addition, we assume that the geometrical mapping $\mathbf{x}$ is invertible a.e. in $\widehat{\Omega}$. By introducing suitable knot vectors $\Xi_{1}$ and $\Xi_{2}$, we define $n_{b f}$ B-spline basis functions $\widehat{N}_{i}(\boldsymbol{\xi})$ in $\widehat{\Omega}$, for $i=1, \ldots, n_{b f}$, by means of the tensor product rule applied to univariate B-spline basis functions; then, by introducing a set of $n_{b f}$ weights, we can derive the corresponding NURBS basis functions $\widehat{R}_{i}(\boldsymbol{\xi})$, for $i=1, \ldots, n_{b f}$. The NURBS domain $\Omega$ is defined by means of the geometrical mapping $\mathbf{x}(\boldsymbol{\xi})=\sum_{i=1}^{n_{b f}} \widehat{R}_{i}(\boldsymbol{\xi}) \mathbf{P}_{i}$, where $\mathbf{P}_{i} \in \mathbb{R}^{3}$, for $i=1, \ldots, n_{b f}$, are the control points. By construction, the properties of the B-spline and NURBS basis functions depend on the knot vectors $\Xi_{1}$ and $\Xi_{2}$, both in terms of the polynomial degree $p$ and global continuity $C^{\alpha}$ in $\widehat{\Omega}$, with $\alpha \geq 0$. In particular, when referring to an univariate B-spline or NURBS basis, the multiplicity $m$ of a knot value determine the local continuity of the basis functions, which is equal to $p-m$. As for example, we report in Fig. 1 some univariate B-spline basis functions of different regularities. We finally remark that the knot vectors define a "mesh" $\widehat{\mathcal{T}}_{h}$ in the parametric domain $\widehat{\Omega}$, comprised of $n_{e l}$ elements $\widehat{\Omega}_{e}$, of which $\widetilde{n}_{e l}$ are of non-zero size; correspondingly, the global mesh size in the parametric domain is indicated as $\widehat{h}$. In the same manner, in virtue of the geometrical mapping $\mathbf{x}$, we define a mesh $\mathcal{T}_{h}$ on the surface $\Omega$, with the associated global mesh size $h$. For a more detailed overview, we refer the interested reader to $[23,24,28,52]$.

IGA is based on the reversal of the standard isoparametric concept [23, 38]; indeed, the same basis functions used for the geometrical representation of the computational domain $\Omega$ are then also used to build the trial function space of the approximate solution of the PDE. With this aim, we define the following finite dimensional NURBS function space on the surface $\Omega$ :

$$
\mathcal{N}_{h}:=\operatorname{span}\left\{R_{i}(\mathbf{x}), i=1, \ldots, n_{b f}\right\},
$$

where $R_{i}(\mathbf{x}):=\widehat{R}_{i}(\boldsymbol{\xi}) \circ \mathbf{x}^{-1}(\boldsymbol{\xi})$, for $i=1 \ldots, n_{b f}$, are the NURBS basis functions in the physical domain $\Omega$. We remark that the function space $\mathcal{N}_{h}$ can be suitably enriched by means of the so-called $h-, p$-, or $k$-refinements procedures, the latter being particular to NURBS, for which both the polynomial degree and the global continuity of the basis functions can be suitably elevated in the computational domain; see e.g. $[23,24,27]$. Then, by referring to the transmembrane potential $v(t)$ in $(6)$ and its spatial approximation, we introduce, with abuse of notation, the following finite dimensional trial space defined on the surface $\Omega$ :

$$
\mathcal{V}_{h}:=\mathcal{V} \cap \mathcal{N}_{h}
$$

for which the approximate transmembrane potential can be written as $v_{h}(t)=\sum_{i=1}^{n_{D O F}^{v}} R_{i}(\mathbf{x}) V_{i}(t)$, being $n_{D O F}^{v}$ the dimension of the function space $\mathcal{V}_{h}$ in terms of the spatial approximation and $\left\{V_{i}(t)\right\}_{i=1}^{n_{D O F}^{v}}$ the set of time dependent control variables; we remark that by reordering the indices of the NURBS basis functions and 
control variables, the full discrete solution in $\mathcal{N}_{h}$ can be rewritten as $v_{h}(t)=\sum_{i=1}^{n_{b f}} R_{i}(\mathbf{x}) V_{i}(t)$, with the vector $\mathbf{v}(t)=\left(V_{1}(t), \ldots, V_{n_{b f}}(t)\right)^{T} \in \mathbb{R}^{n_{b f}}$ storing the control variables. We recall that in the standard Isogeometric approach, including the case of surfaces [28], the weak formulation of the problem is recast into the parametric domain $\widehat{\Omega}$ for which the transmembrane potential $\widehat{v}_{h}(t)$ is an element of $\widehat{\mathcal{N}}_{h}:=\operatorname{span}\left\{\widehat{R}_{i}(\boldsymbol{\xi}), i=1, \ldots, n_{b f}\right\}$ for any given time $t$; after having solved the problem defined in $\widehat{\Omega}$, then we obtain the approximate solution $v_{h}(t)$ on the surface $\Omega$ by using the geometrical mapping $\mathbf{x}(\boldsymbol{\xi})$. Since we use NURBS-based IGA in the framework of the Galerkin method for the spatial approximation of the first equation in problem (6), we introduce the finite dimensional test function space $\mathcal{V}_{0, h}:=\mathcal{V}_{0} \cap \mathcal{N}_{h}$. Specifically, from (6), we obtain:

$$
\begin{aligned}
& \forall t \in(0, T), \text { find } v_{h}(t) \in \mathcal{V}_{h} \quad: \\
& \begin{aligned}
\left(\phi_{h}, C_{m} \frac{d v_{h}}{d t}(t)\right) & +\left(\nabla_{\Omega} \phi_{h}, \mathbf{D} \nabla_{\Omega} v_{h}(t)\right) \\
& +\left(\phi_{h}, i_{\text {ion }}\left(v_{h}(t), \widetilde{w}(t)\right)\right)-\left(\phi_{h}, i_{\text {app }}(t)\right)=0 \quad \forall \phi_{h} \in \mathcal{V}_{0, h},
\end{aligned}
\end{aligned}
$$

with $v_{h}(0)=v_{0, h}$ in $\Omega$, for a prescribed function $\widetilde{w}(t) \in \mathcal{W}$, where $v_{0, h}$ is the $L^{2}$ projection of $v_{0}$ onto the NURBS space $\mathcal{N}_{h}$. Therefore, the discrete form of the spatially approximated potential equation (10) reads:

$$
\forall t \in(0, T), \text { find } \mathbf{v}(t) \in \mathbb{R}^{n_{b f}} \quad:\left\{\begin{aligned}
\mathbf{r}_{v}\left(\frac{d \mathbf{v}}{d t}(t), \mathbf{v}(t) ; \widetilde{w}(t)\right) & =\mathbf{0}, \\
\mathbf{v}_{\mathbf{i}_{D}}(t) & =\mathbf{v}_{D}(t),
\end{aligned}\right.
$$

with $\mathbf{v}(0)=\mathbf{v}_{0}$, where the residual vector $\mathbf{r}_{v}\left(\frac{d \mathbf{v}}{d t}(t), \mathbf{v}(t) ; \widetilde{w}(t)\right) \in \mathbb{R}^{n_{b f}}$ is defined by components as:

$$
\begin{aligned}
\mathbf{r}_{v, i}\left(\frac{d \mathbf{v}}{d t}(t), \mathbf{v}(t) ; \widetilde{w}(t)\right):= & \left(R_{i}, C_{m} \frac{d v_{h}}{d t}(t)\right)+\left(\nabla_{\Omega} R_{i}, \mathbf{D} \nabla_{\Omega} v_{h}(t)\right) \\
& +\left(R_{i}, i_{\text {ion }}\left(v_{h}(t), \widetilde{w}(t)\right)\right)-\left(R_{i}, i_{\text {app }}(t)\right) \quad \text { for } i=1, \ldots, n_{b f},
\end{aligned}
$$

$\mathbf{v}_{0} \in \mathbb{R}^{n_{b f}}$ and $\mathbf{v}_{D}(t) \in \mathbb{R}^{n_{D}}$ are the vectors of the control variables corresponding to $v_{0, h}$ and the essential (Dirichlet) data, respectively, and the vector $\mathbf{i}_{D}$ identifies the $n_{D}$ control variables associated to the essential boundary conditions. We remark that the spatial approximation of (10) involves the computation of integrals corresponding to forms and functionals, for which suitable quadrature formula should be used; while different and computationally efficient possibilities exist for NURBS-based IGA (see e.g. [4]), we use the standard Gauss-Legendre formulas [56] with $(p+1)^{2}$ quadrature nodes per mesh element for a total of $n_{q n}=(p+1)^{2} \widetilde{n}_{e l}$ nodes in $\mathcal{T}_{h}$; in this manner, for a general function $\varphi: \Omega \rightarrow \mathbb{R}$, we have $\int_{\Omega} \varphi(\mathbf{x}) d \Omega \approx \sum_{q=1}^{n_{q n}} \varphi\left(\mathbf{x}_{q}\right) \alpha_{q}$, where $\left\{\mathbf{x}_{q}\right\}_{q=1}^{n_{q n}}$ and $\left\{\alpha_{q}\right\}_{q=1}^{n_{q n}}$ are the quadrature nodes and weights, respectively. For more details regarding the spatial approximation of PDEs on surfaces by means of NURBS-based IGA in the framework of the Galerkin method, we refer the interested reader to [28].

Next we consider the spatial approximation of the recovery variable $\widetilde{w}(t) \in \mathcal{W}$ in (11) and (12) for all $t \in(0, T)$. Two different approaches are typically used and we illustrate them for NURBS basis functions; in 
the following, we outline a brief, formal overview of these approaches within the context of IGA. In the first approach, one looks for an approximate recovery variable $\widetilde{w}_{h}(t) \in \mathcal{W}_{h}$, being $\mathcal{W}_{h} \subset \mathcal{W}$ a finite dimensional function space; this approach is called nodal interpolation (NI) and is typically used with interpolatory basis functions as e.g. for the Finite Element method. More specifically, when considering NURBS basis functions, we can choose $\mathcal{W}_{h}:=\mathcal{W} \cap \mathcal{N}_{h}$, for which the approximate recovery variable reads $\widetilde{w}_{h}(t)=\sum_{i=1}^{n_{b f}} R_{i}(\mathbf{x}) \widetilde{W}_{i}(t)$, being the dimension of the function space $\mathcal{W}_{h}$ generally coincident with that of $\mathcal{N}_{h}$ (i.e. $\left.n_{b f}\right)$ and $\left\{\widetilde{W}_{i}(t)\right\}_{i=1}^{n_{b f}}$ the set of time dependent control variables such that $\widetilde{\mathbf{w}}(t)=\left(W_{1}(t), \ldots, W_{n_{b f}}(t)\right)^{T} \in \mathbb{R}^{n_{b f}}$. We remark that, due to the nature of NURBS basis functions, the approach is not interpolatory as the recovery control variables do not correspond to physical points on the surface $\Omega$. We notice that the approximate recovery variable $\widetilde{w}_{h}(t)$ affects the potential equation (10) through the ionic current term $i_{\text {ion }}\left(v_{h}(t), \widetilde{w}_{h}(t)\right)$, which requires its evaluation at quadrature nodes for the approximation of the integrals. At least two different possibilities have been considered in literature $[43,50]$ for the evaluation of the ionic current at the $n_{n q}$ quadrature nodes of the mesh $\mathcal{T}_{h}$. In the first one, called state variable interpolation (NI-SVI) the potential and recovery variables are evaluated ("interpolated") at the quadrature nodes $\left\{\mathbf{x}_{q}\right\}_{q=1}^{n_{q n}}$, for which the current is computed as:

$$
i_{\mathrm{ion}}^{\mathrm{SVI}}\left(\mathbf{x}_{q}\right)=i_{\text {ion }}\left(\sum_{i=1}^{n_{b f}} R_{i}\left(\mathbf{x}_{q}\right) V_{i}(t), \sum_{i=1}^{n_{b f}} R_{i}\left(\mathbf{x}_{q}\right) \widetilde{W}_{i}(t)\right) .
$$

In the second one, called ionic current interpolation (NI-ICI), the current is first evaluated at the control variables and then "interpolated" at the quadrature nodes by means of the NURBS basis functions as:

$$
i_{\text {ion }}^{\mathrm{ICI}}\left(\mathbf{x}_{q}\right)=\sum_{i=1}^{n_{b f}} R_{i}\left(\mathbf{x}_{q}\right) i_{\text {ion }}\left(V_{i}(t), \widetilde{W}_{i}(t)\right) ;
$$

in this manner, an approximate evaluation of the ionic current is obtained at the quadrature nodes, but the computationally expensive evaluations of the basis functions at the quadrature nodes $\left\{\mathbf{x}_{q}\right\}_{q=1}^{n_{q n}}$ can be computed only once for all $t \in(0, T]$.

The second approach for the spatial approximation of the recovery variable $\widetilde{w}(t)$, called Gauss integration (GI), consists in defining a vector $\overline{\mathbf{w}}(t)=\left(\bar{W}_{1}(t), \ldots, \bar{W}_{n q}(t)\right)^{T} \in \mathbb{R}^{n_{n q}}$ of $n_{q n}$ recovery control variables "ideally" defined correspondingly to the quadrature nodes $\left\{\mathbf{x}_{q}\right\}_{q=1}^{n_{q n}}$ on $\Omega$ for which the ionic current is then evaluated, similarly to the NI-ICI case, as:

$$
i_{\text {ion }}^{\mathrm{GI}}\left(\mathbf{x}_{q}\right)=\sum_{i=1}^{n_{b f}} R_{i}\left(\mathbf{x}_{q}\right) i_{\mathrm{ion}}\left(V_{i}(t), \bar{W}_{q}(t)\right) .
$$

We remark that the approach chosen for the spatial approximation of the recovery variable $w(t)$ may have significant consequences on the accuracy of the solution of the full coupled monodomain problem (7). For example, in [43], the results obtained when considering the Finite Element method with NI-ICI and GI approaches have been compared. Specifically, it was shown that the exact conduction velocity of the 
action potential is underestimated by using the NI-ICI approach and overestimated by the GI one; in both the cases, a significantly refined mesh was needed to obtain accurate approximations of the front velocity. Similar behavior holds for the NI-SVI method [47], as in the case of GI. While we notice that a similar effect can be obtained when considering NURBS-based IGA, we remark that in this paper we are mainly focusing on the study of the effects of the continuity of the NURBS basis functions for a specific approach. For this reason, we selected in this paper a unique approach for the spatial approximation of the recovery variable $w(t)$, specifically the analogous of the NI-ICI approach for NURBS basis functions. Our choice is motivated by the simplicity and efficiency of the numerical implementation of the algorithm in combination with the time discretization approach; in this respect, we remark that in [14], the analogous of the NI-SVI approach is instead used for NURBS-based IGA.

For the NI-ICI approach in the context of NURBS-based IGA, we select the finite dimensional function space

$$
\mathcal{W}_{h}:=\mathcal{W} \cap \mathcal{N}_{h}
$$

as trial space for the approximate recovery variable $w_{h}(t)$. In view of the application of the NI-ICI approach to the problem (11) for some $\widetilde{w}_{h}(t) \in \mathcal{W}_{h}$, we reformulate the ionic current function $i_{\text {ion }}$ as:

$$
\begin{aligned}
& i_{\text {ion }}^{\mathrm{ICI}}(\mathbf{v}(t), \widetilde{\mathbf{w}}(t)):=\sum_{i=1}^{n_{b f}} R_{i} i_{\text {ion }}\left(V_{i}(t), \widetilde{W}_{i}(t)\right), \\
& \mathbf{i}_{\text {ion }}^{\mathrm{ICI}}(\mathbf{v}(t), \widetilde{\mathbf{w}}(t)):=\left(i_{\text {ion }}\left(V_{1}(t), \widetilde{W}_{1}(t)\right), \ldots, i_{\text {ion }}\left(V_{n_{b f}}(t), \widetilde{W}_{n_{b f}}(t)\right)\right)^{T} \in \mathbb{R}^{n_{b f}} .
\end{aligned}
$$

Similarly, we assume that the applied current function $i_{\text {app }}(t)$ can be written in the form:

$$
\begin{aligned}
i_{\mathrm{app}}^{\mathrm{ICI}}(t) & :=\sum_{i=1}^{n_{b f}} R_{i} i_{\mathrm{app}, i}(t), \\
\mathbf{i}_{\mathrm{app}}^{\mathrm{ICI}}(t) & :=\left(i_{\mathrm{app}, 1}(t), \ldots, i_{\mathrm{app}, n_{b f}}(t)\right)^{T} \in \mathbb{R}^{n_{b f}} .
\end{aligned}
$$

In this manner, the NI-ICI spatial discretization of problem (11) reads:

$$
\forall t \in(0, T), \text { find } \mathbf{v}(t) \in \mathbb{R}^{n_{b f}}:\left\{\begin{aligned}
\mathbf{r}_{v}^{\mathrm{ICI}}\left(\frac{d \mathbf{v}}{d t}(t), \mathbf{v}(t) ; \widetilde{\mathbf{w}}(t)\right) & =\mathbf{0}, \\
\mathbf{v}_{\mathbf{i}_{D}}(t) & =\mathbf{v}_{D}(t),
\end{aligned}\right.
$$

with $\mathbf{v}(0)=\mathbf{v}_{0}$, where the residual $\mathbf{r}_{v}^{\mathrm{ICI}}\left(\frac{d \mathbf{v}}{d t}(t), \mathbf{v}(t) ; \widetilde{\mathbf{w}}(t)\right) \in \mathbb{R}^{n_{b f}}$ is defined by components using (14) and (15) as:

$$
\begin{aligned}
\mathbf{r}_{v, i}^{\mathrm{ICI}}\left(\frac{d \mathbf{v}}{d t}(t), \mathbf{v}(t) ; \widetilde{\mathbf{w}}(t)\right):= & \left(R_{i}, C_{m} \frac{d v_{h}}{d t}(t)\right)+\left(\nabla_{\Omega} R_{i}, \mathbf{D} \nabla_{\Omega} v_{h}(t)\right) \\
& +\left(R_{i}, i_{\mathrm{ion}}^{\mathrm{ICI}}(\mathbf{v}(t), \widetilde{\mathbf{w}}(t))\right)-\left(R_{i}, i_{\mathrm{app}}^{\mathrm{ICI}}(t)\right) \quad \text { for } i=1, \ldots, n_{b f} .
\end{aligned}
$$

We remark that, even for $w_{h}(t) \in \mathcal{W}_{h}$, the spatial approximation of the equation for the recovery variable (see the second equation in problem (7)) can be addressed in different ways. By following a 
procedure similar to NI-ICI for the recovery function $g_{\text {rec }}$ and by indicating with $\widetilde{v}_{h}(t)=\sum_{i=1}^{n_{b f}} R_{i}(\mathbf{x}) \widetilde{V}_{i}(t)$ the $L^{2}$ projection onto the NURBS space $\mathcal{N}_{h}$ of a prescribed potential variable $\widetilde{v}(t)$ for all $t \in(0, T)$ and with $\widetilde{\mathbf{v}}(t)=\left(\widetilde{V}_{1}(t), \ldots, \widetilde{V}_{n_{b f}}(t)\right)^{T} \in \mathbb{R}^{n_{b f}}$ the associated vector of control variables, we define:

$$
\begin{aligned}
& g_{\text {rec }}^{\mathrm{ICI}}(\widetilde{\mathbf{v}}(t), \mathbf{w}(t)):=\sum_{i=1}^{n_{b f}} R_{i} g_{\text {rec }}\left(\widetilde{V}_{i}(t), W_{i}(t)\right) \\
& \mathbf{g}_{\text {rec }}^{\mathrm{ICI}}(\widetilde{\mathbf{v}}(t), \mathbf{w}(t)):=\left(g_{\text {rec }}\left(\widetilde{V}_{1}(t), W_{1}(t)\right), \ldots, g_{\text {rec }}\left(\widetilde{V}_{n_{b f}}(t), W_{n_{b f}}(t)\right)\right)^{T} \in \mathbb{R}^{n_{b f}} .
\end{aligned}
$$

Then, we obtain the discrete equation for the recovery variable:

$$
\forall t \in(0, T), \text { find } \mathbf{w}(t) \in \mathbb{R}^{n_{b f}} \quad: \quad \mathbf{r}_{w}^{\mathrm{ICI}}\left(\frac{d \mathbf{w}}{d t}(t), \mathbf{w}(t) ; \widetilde{\mathbf{v}}(t)\right)=\mathbf{0},
$$

with $\mathbf{w}_{h}(0)=\mathbf{w}_{0, h}$, for an assigned potential $\widetilde{v}(t) \in \mathcal{V}$, where the residual vector $\widetilde{\mathbf{r}}_{w}^{\mathrm{ICI}}\left(\frac{d \mathbf{w}}{d t}(t), \mathbf{w}(t) ; \widetilde{\mathbf{v}}(t)\right) \in \mathbb{R}^{n_{b f}}$ is defined using (18) as:

$$
\mathbf{r}_{w}^{\mathrm{ICI}}\left(\frac{d \mathbf{w}}{d t}(t), \mathbf{w}(t) ; \widetilde{\mathbf{v}}(t)\right):=\frac{d \mathbf{w}}{d t}(t)-\mathbf{g}_{\text {rec }}^{\mathrm{ICI}}(\widetilde{\mathbf{v}}(t), \mathbf{w}(t))
$$

and $\mathbf{w}_{0, h}$ is the vector of control variables corresponding to the $L^{2}$ projection of $w_{0}$ onto the NURBS space $\mathcal{N}_{h}$. We remark that problem (19) represents a system of first order ODEs.

Finally, by combining (16) and (19) in a coupled problem, we obtain the full spatial approximation of the monodomain problem (7) by means of NURBS-based IGA, which at the discrete level reads:

$$
\forall t \in(0, T), \text { find } \mathbf{v}(t), \mathbf{w}(t) \in \mathbb{R}^{n_{b f}} \quad:\left\{\begin{aligned}
\mathbf{r}_{v}^{\mathrm{ICI}}\left(\frac{d \mathbf{v}}{d t}(t), \mathbf{v}(t), \mathbf{w}(t)\right) & =\mathbf{0}, \\
\mathbf{v}_{\mathbf{i}_{D}}(t) & =\mathbf{v}_{D}(t), \\
\mathbf{r}_{w}^{\mathrm{ICI}}\left(\frac{d \mathbf{w}}{d t}(t), \mathbf{v}(t), \mathbf{w}(t)\right) & =\mathbf{0},
\end{aligned}\right.
$$

with $\mathbf{v}(0)=\mathbf{v}_{0}$ and $\mathbf{w}(0)=\mathbf{w}_{0}$, where the residuals are defined following Eqs. (17) and (20) as:

$$
\begin{aligned}
\mathbf{r}_{v, i}^{\mathrm{ICI}}\left(\frac{d \mathbf{v}}{d t}(t), \mathbf{v}(t), \mathbf{w}(t)\right):= & \left(R_{i}, C_{m} \frac{d v_{h}}{d t}(t)\right)+\left(\nabla_{\Omega} R_{i}, \mathbf{D} \nabla_{\Omega} v_{h}(t)\right) \\
& +\left(R_{i}, i_{\text {ion }}^{\mathrm{ICI}}(\mathbf{v}(t), \mathbf{w}(t))\right)-\left(R_{i}, i_{\mathrm{app}}^{\mathrm{ICI}}(t)\right) \quad \text { for } i=1, \ldots, n_{b f}, \\
\mathbf{r}_{w}^{\mathrm{ICI}}\left(\frac{d \mathbf{w}}{d t}(t), \mathbf{w}(t), \mathbf{v}(t)\right):= & \frac{d \mathbf{w}}{d t}(t)-\mathbf{g}_{\text {rec }}^{\mathrm{ICI}}(\mathbf{v}(t), \mathbf{w}(t)) .
\end{aligned}
$$

\subsection{Time discretization}

We consider now the time discretization of the monodomain problem (21) spatially approximated by NURBS-based IGA; specifically, we adopt the second order Strang's symmetrical splitting scheme [41] based on the separation of the diffusive and reaction (zero order) terms in the system of ODEs correspondingly to 
the potential equation (16), which appears in the first equation of problem (21). By considering directly the first residual in Eq. (22), we can identify the following (split) residuals in view of using the splitting scheme:

$$
\begin{aligned}
\mathbf{r}_{v, \mathrm{diff}, i}^{\mathrm{ICI}}\left(\frac{d \mathbf{v}}{d t}(t), \mathbf{v}(t)\right) & :=\left(R_{i}, C_{m} \frac{d v_{h}}{d t}(t)\right)+\left(\nabla_{\Omega} R_{i}, \mathbf{D} \nabla_{\Omega} v_{h}(t)\right) \quad \text { for } i=1, \ldots, n_{b f}, \\
\mathbf{r}_{v, \text { react }}^{\mathrm{ICI}}\left(\frac{d \mathbf{v}}{d t}(t), \mathbf{v}(t), \mathbf{w}(t)\right) & :=C_{m} \frac{d \mathbf{v}}{d t}(t)+\mathbf{i}_{\mathrm{ion}}^{\mathrm{ICI}}(\mathbf{v}(t), \mathbf{w}(t))-\mathbf{i}_{\text {app }}^{\mathrm{ICI}}(t) ;
\end{aligned}
$$

the latter has been obtained by rewriting the zero order terms in strong form, as allowed for the NI-ICI approach under consideration. The residual vector $\mathbf{r}_{v, \text { diff }}^{\mathrm{ICI}}\left(\frac{d \mathbf{v}}{d t}(t), \mathbf{v}(t)\right)$ can be also rewritten as:

$$
\mathbf{r}_{v, \text { diff }}^{\text {ICI }}\left(\frac{d \mathbf{v}}{d t}(t), \mathbf{v}(t)\right)=\mathbf{M}_{C_{m}} \frac{d \mathbf{v}}{d t}(t)+\mathbf{K}_{\mathbf{D}} \mathbf{v}(t),
$$

where, by virtue of the IGA spatial approximation, $\mathbf{M}_{C_{m}}$ and $\mathbf{K}_{\mathbf{D}} \in \mathbb{R}^{n_{b f} \times n_{b f}}$, with $\left(\mathbf{M}_{C_{m}}\right)_{i j}:=\left(R_{i}, C_{m} R_{j}\right)$ and $\left(\mathbf{K}_{\mathbf{D}}\right)_{i j}:=\left(\nabla_{\Omega} R_{i}, \mathbf{D} \nabla_{\Omega} R_{j}\right)$, for $i, j=1, \ldots, n_{b f}$.

Let us partition the time interval $(0, T)$ into $N_{t}$ times steps of size $\Delta t=T / N_{t}$, yielding the discrete times $t_{k}=k \Delta t$, for $k=0, \ldots, N_{t}$. Then, we introduce the time dependent vectors $\mathbf{v}^{k,(1)}(t)$ and $\mathbf{w}^{k,(1)}(t) \in \mathbb{R}^{n_{b f}}$ defined for $t \in\left(t_{k}, t_{k+1 / 2}\right), \mathbf{v}^{k,(2)}(t) \in \mathbb{R}^{n_{b f}}$ defined for $t \in\left(t_{k}, t_{k+1}\right)$, and finally $\mathbf{v}^{k,(3)}(t)$ and $\mathbf{w}^{k,(3)}(t) \in \mathbb{R}^{n_{b f}}$ defined for $t \in\left(t_{k+1 / 2}, t_{k+1}\right)$, for any $k=0, \ldots, N_{t}-1$ with $t_{k+1 / 2}:=t_{k}+\Delta t / 2$. In this manner, we outline the following splitting scheme at the generic discrete time $t_{k}$, for $k=0, \ldots, N_{t}-1$, as the sequential solution of the following three problems:

$$
\forall t \in\left(t_{k}, t_{k+1 / 2}\right), \text { find } \mathbf{v}^{k,(1)}(t), \mathbf{w}^{k,(1)}(t) \in \mathbb{R}^{n_{b f}}:\left\{\begin{array}{c}
\mathbf{r}_{v, \text { react }}^{\mathrm{ICI}}\left(\frac{d \mathbf{v}^{k,(1)}}{d t}(t), \mathbf{v}^{k,(1)}(t), \mathbf{w}^{k,(1)}(t)\right)=\mathbf{0} \\
\mathbf{r}_{w}^{\mathrm{ICI}}\left(\frac{d \mathbf{w}^{k,(1)}}{d t}(t), \mathbf{v}^{k,(1)}(t), \mathbf{w}^{k,(1)}(t)\right)=\mathbf{0}
\end{array}\right.
$$

with $\mathbf{v}^{k,(1)}\left(t_{k}\right)=\mathbf{v}_{0}^{k,(1)}$ and $\mathbf{w}^{k,(1)}\left(t_{k}\right)=\mathbf{w}_{0}^{k,(1)}$

$$
\forall t \in\left(t_{k}, t_{k+1}\right), \text { find } \mathbf{v}^{k,(2)}(t) \in \mathbb{R}^{n_{b f}}:\left\{\begin{aligned}
\mathbf{r}_{v, \text { diff }}^{\mathrm{ICI}}\left(\frac{d \mathbf{v}^{k,(2)}}{d t}(t), \mathbf{v}^{k,(2)}(t)\right) & =\mathbf{0} \\
\mathbf{v}_{\mathbf{i}_{D}}^{k,(2)}(t) & =\mathbf{v}_{D}(t)
\end{aligned}\right.
$$

with $\mathbf{v}^{k,(2)}\left(t_{k}\right)=\mathbf{v}^{k,(1)}\left(t_{k+1 / 2}\right)$

$$
\forall t \in\left(t_{k+1 / 2}, t_{k+1}\right), \text { find } \mathbf{v}^{k,(3)}(t), \mathbf{w}^{k,(3)}(t) \in \mathbb{R}^{n_{b f}}:\left\{\begin{array}{c}
\mathbf{r}_{v, \text { react }}^{\text {ICI }}\left(\frac{d \mathbf{v}^{k,(3)}}{d t}(t), \mathbf{v}^{k,(3)}(t), \mathbf{w}^{k,(3)}(t)\right)=\mathbf{0} \\
\mathbf{r}_{w}^{\text {ICI }}\left(\frac{d \mathbf{w}^{k,(3)}}{d t}(t), \mathbf{v}^{k,(3)}(t), \mathbf{w}^{k,(3)}(t)\right)=\mathbf{0}
\end{array}\right.
$$

with $\mathbf{v}^{k,(3)}\left(t_{k+1 / 2}\right)=\mathbf{v}^{k,(2)}\left(t_{k+1}\right)$ and $\mathbf{w}^{k,(3)}\left(t_{k+1 / 2}\right)=\mathbf{w}^{k,(1)}\left(t_{k+1 / 2}\right)$, where $\mathbf{v}_{0}^{k,(1)}:=\mathbf{v}^{k,(3)}\left(t_{k}\right)$ and $\mathbf{w}_{0}^{k,(1)}:=\mathbf{w}^{k,(3)}\left(t_{k}\right)$ for $k=1, \ldots, N_{t}-1$, while $\mathbf{v}_{0}^{k,(1)}:=\mathbf{v}_{0}$ and $\mathbf{w}_{0}^{k,(1)}:=\mathbf{w}_{0}$ for $k=0$.

We remark that problems (25), (26), and (27) are still continuously dependent on the time variable $t \in\left(t_{k}, t_{k+1}\right)$ for $k=0, \ldots, N_{t}-1$ and need to be fully discretized in time. With this aim, we consider 
the backward Euler method for problems (25) and (27), while the generalized- $\alpha$ method (see [17] and e.g. [45]) for problem (26). The latter is an implicit, unconditionally absolutely stable method with control on high frequency dissipation, which, for a linear problem, is also second order accurate. In view of using such discretization schemes, we indicate with $\mathbf{v}_{k}^{(1)}, \mathbf{v}_{k+1 / 2}^{(1)}, \mathbf{w}_{k}^{(1)}, \mathbf{w}_{k+1 / 2}^{(1)}, \mathbf{v}_{k}^{(2)}, \mathbf{v}_{k+1}^{(2)}, \mathbf{v}_{k+1 / 2}^{(3)}, \mathbf{v}_{k+1}^{(3)}, \mathbf{w}_{k+1 / 2}^{(3)}$, and $\mathbf{w}_{k+1}^{(3)} \in \mathbb{R}^{n_{b f}}$ the approximations of $\mathbf{v}^{k,(1)}\left(t_{k}\right), \mathbf{v}^{k,(1)}\left(t_{k+1 / 2}\right), \mathbf{w}^{k,(1)}\left(t_{k}\right), \mathbf{w}^{k,(1)}\left(t_{k+1 / 2}\right), \mathbf{v}^{k,(2)}\left(t_{k}\right)$, $\mathbf{v}^{k,(2)}\left(t_{k+1}\right), \mathbf{v}^{k,(3)}\left(t_{k+1 / 2}\right), \mathbf{v}^{k,(3)}\left(t_{k+1}\right), \mathbf{w}^{k,(3)}\left(t_{k+1 / 2}\right)$, and $\mathbf{w}^{k,(3)}\left(t_{k+1}\right) \in \mathbb{R}^{n_{b f}}$, respectively. Then, we recall the parameters $\alpha_{m}=\frac{1}{2}\left(\frac{3-\rho_{\infty}}{1+\rho_{\infty}}\right), \alpha_{f}=\delta=\frac{1}{1+\rho_{\infty}}$ for the generalized- $\alpha$ method, which are dependent on the parameter $\rho_{\infty} \in[0,1]$ controlling the high frequency dissipation, which we set in this work as $\rho_{\infty}=\frac{1}{2}$; this choice is rather common in literature, see e.g. [2, 33, 45], as it allows to damp the high frequencies introduced by the numerical discretization while preserving most of the natural ones associated to the continuous model. Finally, we introduce the auxiliary variables $\mathbf{v}_{k+\alpha_{f}}^{(2)}, \dot{\mathbf{v}}_{k+1}^{(2)}$, and $\dot{\mathbf{v}}_{k+\alpha_{m}}^{(2)} \in \mathbb{R}^{n_{b f}}$ used for the approximation of $\mathbf{v}_{k+1}^{(2)}$ with the generalized- $\alpha$ method and the discrete time $t_{k+\alpha_{f}}=t_{k}+\alpha_{f} \Delta t$. In this manner, the fully discrete splitting scheme at the generic discrete time $t_{k}$, for $k=0, \ldots, N_{t}-1$, corresponds to the consecutive solution of the following three problems:

$$
\text { find } \mathbf{v}_{k+1 / 2}^{(1)}, \mathbf{w}_{k+1 / 2}^{(1)}(t) \in \mathbb{R}^{n_{b f}}:\left\{\begin{array}{c}
\frac{C_{m}}{\Delta t / 2}\left(\mathbf{v}_{k+1 / 2}^{(1)}-\overline{\mathbf{v}}_{k}^{(1)}\right)+\mathbf{i}_{\text {ion }}^{\text {ICI }}\left(\overline{\mathbf{v}}_{k}^{(1)}, \overline{\mathbf{w}}_{k}^{(1)}\right)-\mathbf{i}_{\text {app }}^{\text {ICI }}\left(t_{k}\right)=\mathbf{0} \\
\frac{1}{\Delta t / 2}\left(\mathbf{w}_{k+1 / 2}^{(1)}-\overline{\mathbf{w}}_{k}^{(1)}\right)-\mathbf{g}_{\text {rec }}^{\text {ICI }}\left(\overline{\mathbf{v}}_{k}^{(1)}, \overline{\mathbf{w}}_{k}^{(1)}\right)=\mathbf{0}
\end{array}\right.
$$

find $\mathbf{v}_{k+1}^{(2)}, \mathbf{v}_{k+\alpha_{f}}^{(2)}, \dot{\mathbf{v}}_{k+1}^{(2)}, \dot{\mathbf{v}}_{k+\alpha_{m}}^{(2)} \in \mathbb{R}^{n_{b f}} \quad:$

$$
\begin{aligned}
& \left\{\begin{aligned}
& \mathbf{M}_{C_{m}} \dot{\mathbf{v}}_{k+\alpha_{m}}^{(2)}+\mathbf{K}_{\mathbf{D}} \mathbf{v}_{k+\alpha_{f}}^{(2)}=\mathbf{0} \\
& \mathbf{v}_{k+\alpha_{f}, \mathbf{i}_{D}}^{(2)}=\mathbf{v}_{D}\left(t_{k+\alpha_{f}}\right), \\
& \mathbf{v}_{k+1}^{(2)}=\mathbf{v}_{k+1 / 2}^{(1)}+\Delta t\left[\delta \dot{\mathbf{v}}_{k+1}^{(2)}+(1-\delta) \frac{1}{\Delta t / 2}\left(\mathbf{v}_{k+1 / 2}^{(1)}-\mathbf{v}_{k}^{(1)}\right)\right], \\
& \mathbf{v}_{k+\alpha_{f}}^{(2)}=\alpha_{f} \mathbf{v}_{k+1}^{(2)}+\left(1-\alpha_{f}\right) \mathbf{v}_{k+1 / 2}^{(1)}, \\
& \dot{\mathbf{v}}_{k+\alpha_{m}}^{(2)}=\alpha_{m} \dot{\mathbf{v}}_{k+1}^{(2)}+\left(1-\alpha_{m}\right) \frac{1}{\Delta t / 2}\left(\mathbf{v}_{k+1 / 2}^{(1)}-\mathbf{v}_{k}^{(1)}\right), \\
& \text { find } \mathbf{v}_{k+1}^{(3)}, \mathbf{w}_{k+1}^{(3)}(t) \in \mathbb{R}^{n_{b f}} \quad: \quad\left\{\begin{array}{c}
C_{m} \\
\Delta t / 2
\end{array}\right.\left(\mathbf{v}_{k+1}^{(3)}-\mathbf{v}_{k+1}^{(2)}\right)+\mathbf{i}_{\text {ion }}^{\text {ICI }}\left(\mathbf{v}_{k+1}^{(2)}, \mathbf{w}_{k+1 / 2}^{(1)}\right)-\mathbf{i}_{\text {app }}^{\text {ICI }}\left(t_{k+1 / 2}\right)=\mathbf{0}, \\
& \frac{1}{\Delta t / 2}\left(\mathbf{w}_{k+1}^{(3)}-\mathbf{w}_{k+1 / 2}^{(1)}\right)-\mathbf{g}_{\text {rec }}^{\text {ICI }}\left(\mathbf{v}_{k+1}^{(2)}, \mathbf{w}_{k+1 / 2}^{(1)}\right)=\mathbf{0}
\end{aligned}\right.
\end{aligned}
$$

where we set $\overline{\mathbf{v}}_{k}^{(1)}=\mathbf{v}_{k}^{(3)}$ and $\overline{\mathbf{w}}_{k}^{(1)}=\mathbf{w}_{k}^{(3)}$ for $k=1, \ldots, N_{t}-1$, while $\overline{\mathbf{v}}_{0}^{(1)}=\mathbf{v}_{0}$ and $\overline{\mathbf{w}}_{0}^{(1)}=\mathbf{w}_{0}$ for $k=0$. Finally, according to the splitting method used, the approximations of $\mathbf{v}\left(t_{k}\right)$ and $\mathbf{w}\left(t_{k}\right)$ at the discrete time $t_{k}$, for $k=1, \ldots, N_{t}$, correspond to $\mathbf{v}_{k}=\mathbf{v}_{k}^{(3)}$ and $\mathbf{w}_{k}=\mathbf{w}_{k}^{(3)}$, respectively, with the initial data $\mathbf{v}_{0}$ and $\mathbf{w}_{0}$ provided at $k=0$. 


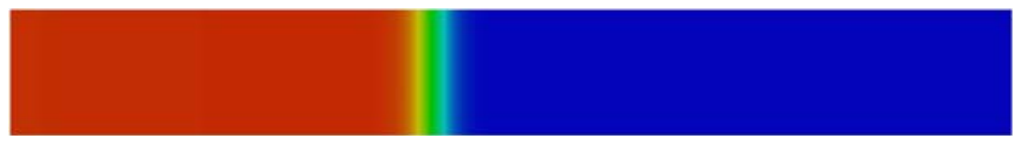

Figure 2: Visualization of the transmembrane potential $v$ (dimensionless) computed for the Mitchel-Schaeffer model at the time $t=25.0 \mathrm{~ms}$ by using a mesh of size $h=1 / 64$ with B-spline basis functions of degree $p=2$ and $C^{1}$-continuous; the number of basis functions is $n_{b f}=2,340$.

\section{Convergence and accuracy study of the IGA spatial approximation of the monodomain equation}

In this section we aim at highlighting the advantage of using high order continuous B-spline basis functions in solving the system (7), approximated as in Eqs. (28)-(30), over their $C^{0}$-continuous counterpart of degree $p$. In particular, we focus on the study of two numerical aspects: the approximation of the conduction velocity of the transmembrane potential fronts and the simulation of spiral wave tip motion.

\subsection{Conduction velocity for the Mitchell-Schaeffer and Aliev-Panfilov monodomain models}

For both the Mitchell-Schaeffer and Aliev-Panfilov models we consider a computational domain $\Omega=$ $(0,2) \times(0,0.25) \mathrm{cm}$ (dimensionless for the Aliev-Panfilov model) and null initial values for dimensionless transmembrane potential $v$ and recovery $w$ variables $v_{0}=0.0$ and $w_{0}=0.0$. The stimulus for the depolarization is applied at the discrete level at time $T_{s}$ by forcing the control variables $\mathbf{v}_{k+1 / 2}^{(1)}, \mathbf{w}_{k+1 / 2}^{(1)}, \mathbf{v}_{k+1}^{(3)}$, and $\mathbf{w}_{k+1}^{(3)}$ associated to the outermost left control points to values allowing the potential fronts to travel towards the right side of $\Omega$. Such numerical stimulus plays the role of the applied current $i_{\text {app }}(t)$ in Eq. (6). We consider different IGA spatial discretizations associated to B-spline basis functions of $\operatorname{degree} p$ and $C^{k}$ continuity, with $k=0$ or $p-1$; the time discretization uses the time step size $\Delta t=0.0025$ ms (dimensionless for the Aliev-Panfilov model).

We start by solving the monodomain equation with the Mitchell-Schaeffer model by setting the following data in Eqs. (3) and (6): $C_{m}=1.00, \mathbb{D}=D_{i s o} \mathbf{I}$, with $\mathbf{I}$ the second order identity tensor and $D=10^{-3} \frac{\mathrm{cm}^{2}}{\mathrm{~ms}}$, $\tau_{\text {in }}=0.300 \mathrm{~ms}, \tau_{\text {out }}=6.00 \mathrm{~ms}, \tau_{\text {open }}=120 \mathrm{~ms}, \tau_{\text {close }}=150 \mathrm{~ms}$, and $v_{\text {rec }}=0.13$; we run the simulation for $T=35.0 \mathrm{~ms}$ and the stimulus is applied up to $T_{s}=1.00 \mathrm{~ms}$. In this manner, $t$ assumes the dimension of ms, the recovery variable $w$ and the potential $v$ are dimensionless; the dimensional counterpart of $v$ can be recovered as $\bar{v}=V_{\min }+\left(V_{\max }-V_{\min }\right) v$, with $V_{\min }=-70.0 \mathrm{mV}$ and $V_{\max }=30.0 \mathrm{mV}$. In Fig. 2 we report the transmembrane potential $v$ computed at $t=25.0 \mathrm{~ms}$ with B-splines over a mesh of size $h=1 / 64$, degree $p=2$, and $C^{1}$-continuous for which $n_{b f}=2,340$; such solution exhibits a front of the potential propagating rightwards. In order to measure the quality of the computation of such front velocity, we compute the mean conduction velocity $V_{f}$ by measuring the distance of the potential front covered over the last $10.0 \mathrm{~ms}$ of the simulation; the distance corresponds to the difference between the averaged coordinates of the contourlines of the potential $v=0.5$ at the times $t=25.0$ and $35.0 \mathrm{~ms}$. We assume that the conduction velocity computed with B-spline basis functions of degree $p=3$ and $C^{2}$-continuous over a mesh of size $h=1 / 320$ represents 


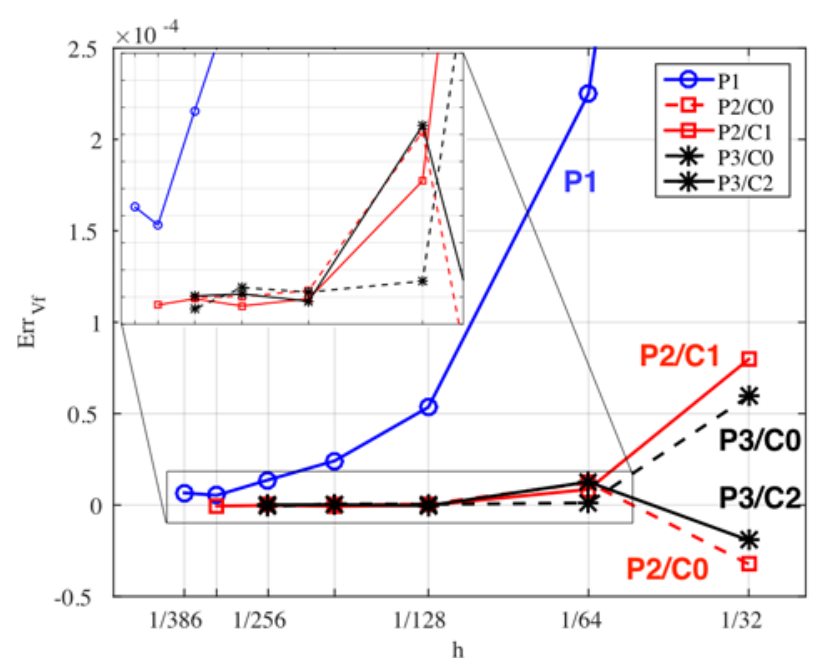

$E_{V_{f}}$ vs. $h$

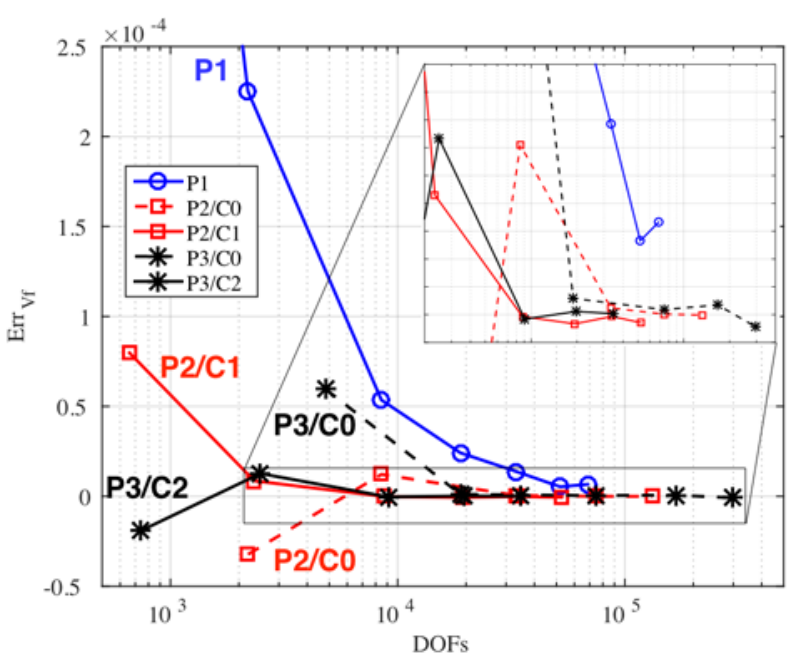

$E_{V_{f}}$ vs. $n_{b f}$

Figure 3: Errors $E_{V_{f}}([\mathrm{~cm} / \mathrm{ms}])$ of the front propagation velocity for the Mitchell-Schaeffer model vs. the mesh size $h$ (left) and the number of B-spline basis functions $n_{b f}$ (right) of degrees $p=1(\circ), 2(\square)$, and $3(*)$ and $C^{0}(--)$ and $C^{p-1}$-continuous (-).

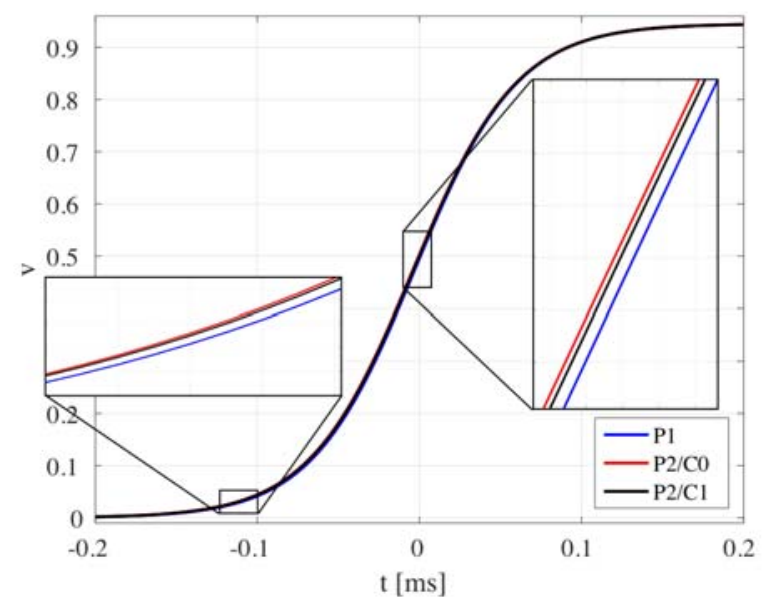

Figure 4: Visualization of the action potential $v$ (dimensionless) computed for the Mitchell-Schaeffer model at the point $(0.5,0.125) \mathrm{cm}$ using B-splines basis functions of degrees $p=1$ (blue) and 2 , the latter both $C^{0}$ (red) and $C^{1}$-continuous (black); the associated numbers of basis functions are $n_{b f}=2,193,2,193$, and 2,340, while the mesh sizes are $h=1 / 64,1 / 32$, and $1 / 64$, respectively. The time axis is shifted for best matching the waveforms at the value $v=0.5$.

the "overkill" value, which we indicate with $V_{f}^{*}=3.3502227 \cdot 10^{-2} \mathrm{~cm} / \mathrm{ms}$. Then, we compute the front velocities $V_{f}$ for different B-spline basis functions of degrees $p=1,2$, and 3 which are $C^{k}$-continuous, with $k=0$ or $p-1$; the mesh sizes $h$ used in the computations range from $h=1 / 32$ down to $1 / 368$ for B-splines of degree $p=1$. The corresponding errors, say $E_{V_{f}}:=V_{f}-V_{f}^{*}$, are displayed in Fig. 3 vs. the mesh size $h$ and the number of basis functions $n_{b f}$. Except for the B-splines of degree $p=1$, those of degrees $p=2$ and 3 deliver accurate results already for relatively coarse meshes, regardless of the continuity, $C^{0}$ or $C^{p-1}$, of the basis functions. Moreover, the higher is the degree $p$ for $C^{0}$-continuous B-spline basis functions, the more accurate is the computed value of $V_{f}$ for a given number of basis functions $n_{b f}$. However, the smoother, 


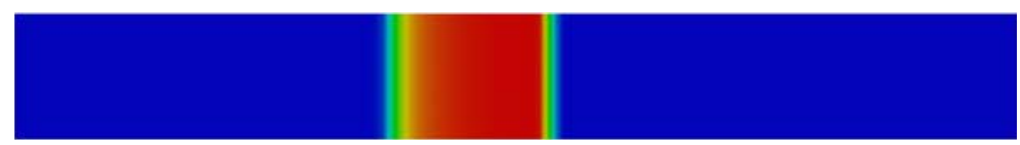

Figure 5: Visualization of the transmembrane potential $v$ (dimensionless) computed for the Aliev-Panfilov model at the dimensionless time $t=80.0$, by using a mesh of size $h=1 / 64$ with B-spline basis functions of degree $p=2$ and $C^{1}$-continuous; the number of basis functions is $n_{b f}=2,340$.

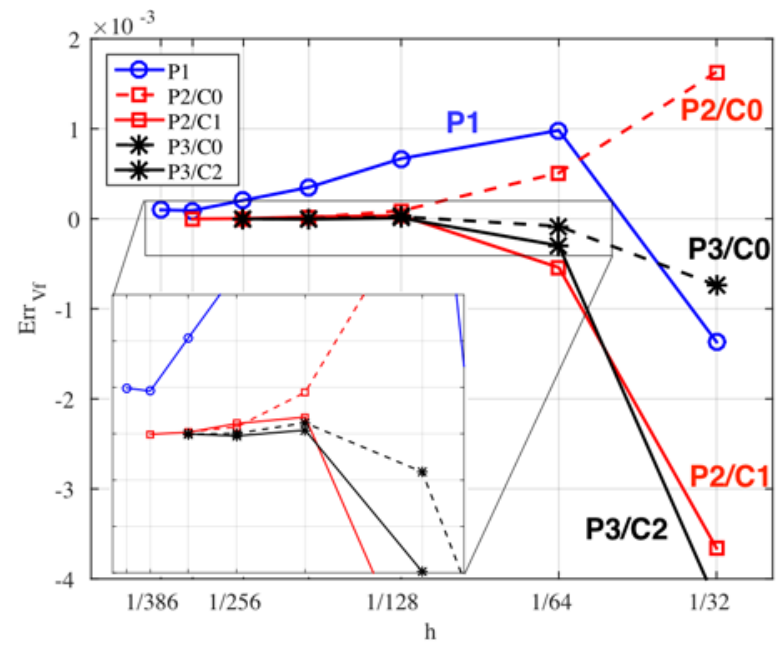

$E_{V_{f}}$ vs. $h$

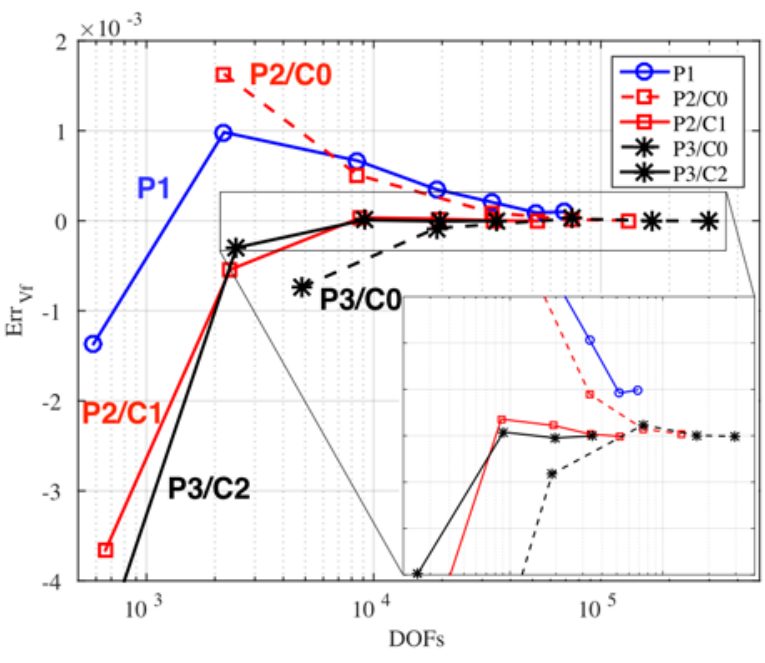

$E_{V_{f}}$ vs. $n_{b f}$

Figure 6: Errors $E_{V_{f}}$ (dimensionless) of front propagation velocity for the Aliev-Panfilov model vs. the mesh size $h$ (left) and number of B-spline basis functions $n_{b f}$ (right) of degrees $p=1(\circ), 2(\square)$, and $3(*)$ and $C^{0}(--)$ and $C^{p-1}$-continuous (-).

globally $C^{p-1}$-continuous B-splines of degrees $p=2$ and 3 deliver much more accurate computations of $V_{f}$ than their $C^{0}$-continuous counterparts, already for relatively small values of $n_{b f}$. Finally, we report in Fig. 4 the evolution of the action potential evaluated in a point of the domain for different choices of the B-splines basis functions; as highlighted, the waveforms of the action potentials are qualitatively very similar already for coarse spatial discretizations.

We solve now the monodomain problem with the Aliev-Panfilov model by setting in Eqs. (4) and (6): $C_{m}=1.00, \mathbb{D}=D_{i s o} \mathbf{I}$ with $D=10^{-4}, k=8.00, a=0.15, \epsilon_{0}=2.00 \cdot 10^{-3}, \mu_{1}=0.200$, and $\mu_{2}=0.300$; we run the simulation for $T=100$ and we apply the initial stimulus up to $T_{s}=0.500$. All the data, $t, w$, and $v$ are dimensionless; the dimensional counterpart of $v$ can be recovered as $\bar{v}=V_{\min }+\left(V_{\max }-V_{\min }\right) v$, with $V_{\min }=-80.0 \mathrm{mV}$ and $V_{\max }=20.0 \mathrm{mV}$, while $\bar{t}=12.9 \mathrm{~ms}$. In Fig. 5 we report as example the transmembrane potential $v$ computed at $t=80.0$ with B-spline basis functions of degree $p=2$ which are $C^{1}$-continuous. As done for the Mitchell-Schaeffer model, we quantitatively compare the results obtained by different B-spline bases in terms of the conduction velocity $V_{f}$ (the one associated to the depolarization front); the mean $V_{f}$ is computed over the last 10.0 dimensionless time units of the simulation. By assuming that the "overkill" front velocity $V_{f}^{*}=1.368069 \cdot 10^{-2}$ is computed with B-splines of degree $p=3$ and 


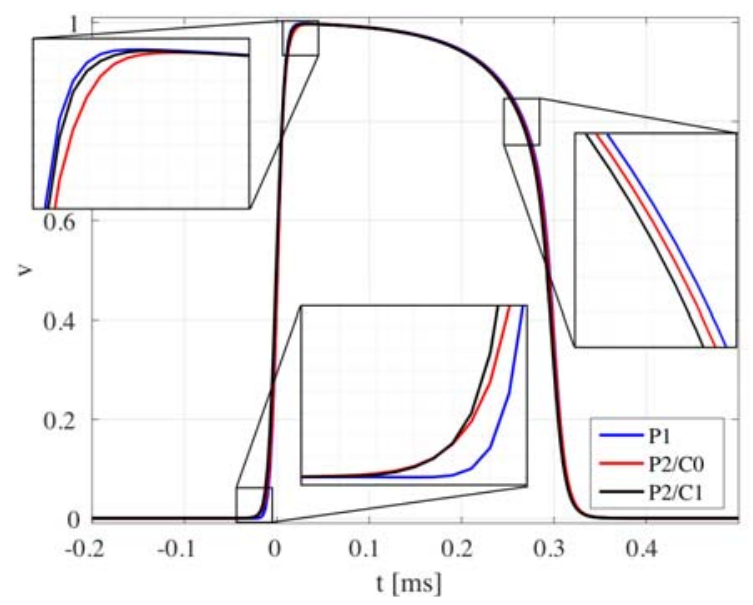

Figure 7: Visualization of the action potential $v$ (dimensionless) computed for the Aliev-Panfilov model at the point $(0.5,0.125)$ using B-splines basis functions of degrees $p=1$ (blue) and 2, the latter both $C^{0}$ (red) and $C^{1}$-continuous (black); the associated numbers of basis functions are $n_{b f}=2,193,2,193$, and 2,340, while the mesh sizes are $h=1 / 64,1 / 32$, and $1 / 64$, respectively. The time axis is shifted for best matching the waveforms at the earliest incurrence of the value $v=0.5$.

$C^{2}$-continuous over a mesh of size $h=1 / 320$, we display the front velocity errors $E_{V_{f}}:=V_{f}-V_{f}^{*}$ associated to the different B-spline bases in Fig. 6. Similarly to the Mitchell-Schaeffer model, the front velocity $V_{f}$ associated to the Aliev-Panfilov model is better captured by B-spline bases of degrees $p=2$ and 3 with respect to degree $p=1$. Moreover, the errors $E_{V_{f}}$ for bases of degrees $p=2$ and 3 are very similar for the same mesh sizes $h$, regardless of the continuity, $C^{0}$ or $C^{p-1}$, of the basis functions. Conversely, in terms of $E_{V_{f}}$ vs. $n_{b f}$, the use of B-splines degrees $p=2$ and $p=3$ is potentially more efficient than for degree $p=1$. Moreover, the use of smooth $C^{p-1}$-continuous B-spline bases of degree $p=2$ and 3 is more accurate than their $C^{0}$-continuous counterpart. Finally, we report in Fig. 7 the evolution of the action potential for different B-splines bases; similarly to the Mitchell-Schaeffer model, the waveforms of the action potentials qualitatively match already for coarse spatial discretizations.

We conclude that, for both the Mitchell-Schaeffer and Aliev-Panfilov models, which develop smooth but sharp and thin interfaces between the polarization and depolarization phases of the transmembrane potential $v$, the use of high order, globally $C^{p-1}$-continuous B-spline basis functions of degree $p$ is more accurate than their $C^{0}$-counterpart. Since the $C^{0}$-continuous B-splines share similar properties with the Lagrangian polynomial bases used in the Finite Element method [38, 54], we speculate that IGA with high order continuous B-splines and NURBS is more accurate and can be more efficient than its Finite Element counterpart, independently of the geometrical advantages in the representation of the computational domain allowed by IGA; even if we only showed numerical results for B-splines of degrees $p=2$ and 3 , the advantages of using globally $C^{p-1}$-continuous basis functions hold also for higher degrees $p \geq 4$. Furthermore, we remark that B-splines and NURBS bases, being positive definite and not interpolatory, as well as endowed with the so-called "variation diminishing property" [23], mitigate the over- and undershooting behavior often occurring with Lagrangian polynomial basis functions in the presence of sharp internal and boundary layers. 


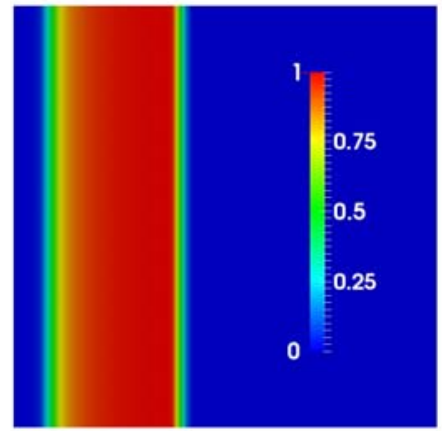

$t=15.0$

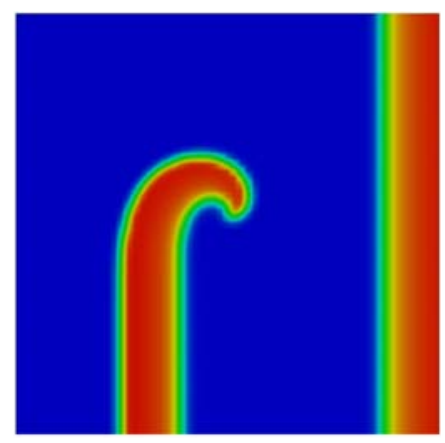

$t=45.0$

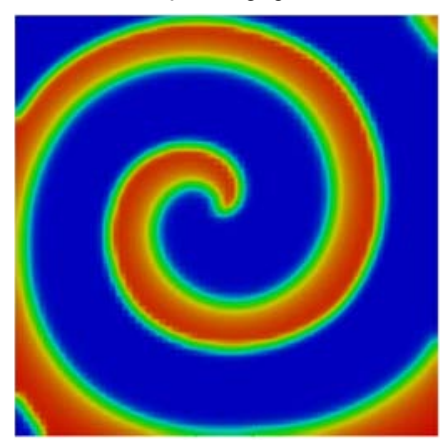

$t=75.0$

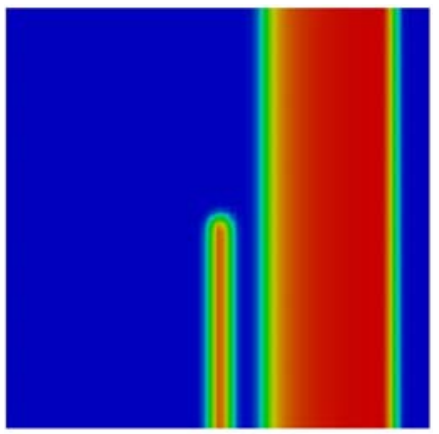

$t=35.1$

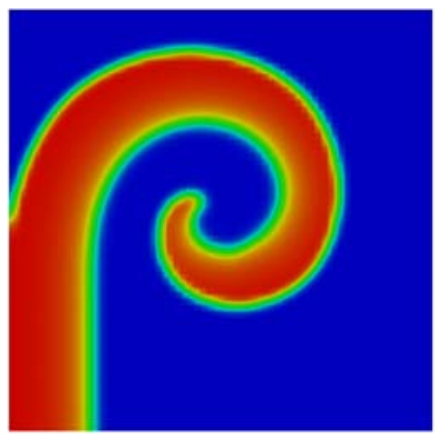

$t=55.0$

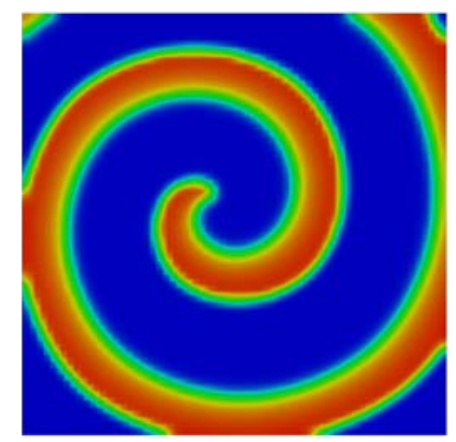

$t=85.0$

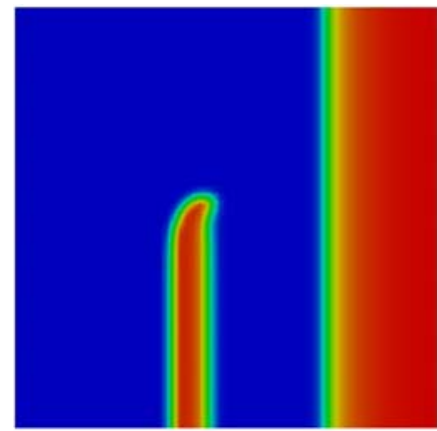

$t=40.0$

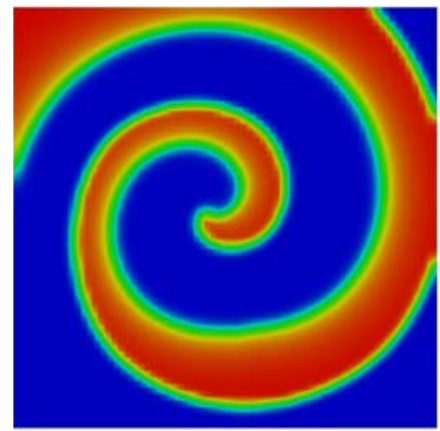

$t=65.0$

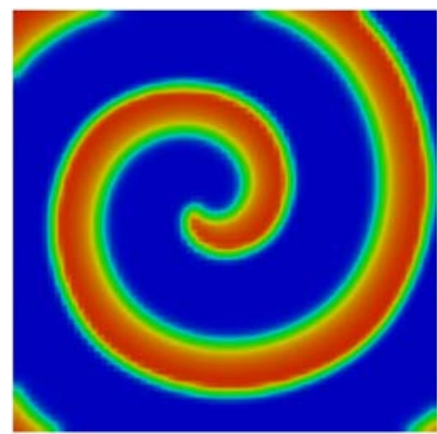

$t=95.0$

Figure 8: Spiral waves of the transmembrane potential induced for the Aliev-Panfilov model at different dimensionless times $t$ computed with B-spline basis functions of degree $p=2$ and $C^{1}$-continuous using a mesh of size $h=1 / 64$ and comprised of $n_{b f}=4,356$.

\subsection{Approximation of spiral waves for the Aliev-Panfilov model}

We now consider the accuracy of the representation of the spiral waves for the monodomain equation with the Aliev-Panfilov model; specifically, we discuss the role of the IGA spatial approximation in the presence of complex solutions for the transmembrane potential $v$. Indeed, the Aliev-Panfilov model [3] can be suitably used to describe patterns of the potential $v$ which are more complicated than the simple unidirectionally propagating pulses considered in Sec. 3.1. As a matter of fact, complex potential fields $v$, as those showing re-entrant spiral or scroll waves, may correspond to pathological diseases of the heart; for example, cardiac arrhythmias can be related to the presence of wavefront spirals which lead to an irregular contraction of the 


\begin{tabular}{c|c|cc|cc} 
B-spline & $p=1$, & $p=2$, & $p=2$, & $p=3$, & $p=3$, \\
basis & $C^{0}$-cont. & $C^{0}$-cont. & $C^{1}$-cont. & $C^{0}$-cont. & $C^{2}$-cont. \\
\hline$n_{e l}$ & 4,096 & 1,024 & 4,096 & 484 & 4,096 \\
$h$ & $1 / 64$ & $1 / 32$ & $1 / 64$ & $1 / 22$ & $1 / 64$ \\
$n_{b f}$ & 4,225 & 4,225 & 4,356 & 4,489 & 4,489
\end{tabular}

Table 1: B-spline bases used for the IGA spatial approximation of the spiral waves associated to the Aliev-Panfilov model with corresponding number of mesh elements $n_{e l}$, mesh sizes $h$, and basis functions $n_{b f}$ used for the space $\mathcal{N}_{h}$.

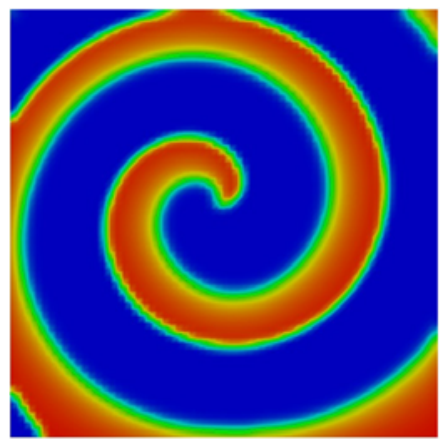

$t=75.0$ $p=1, C^{0}$-continuous

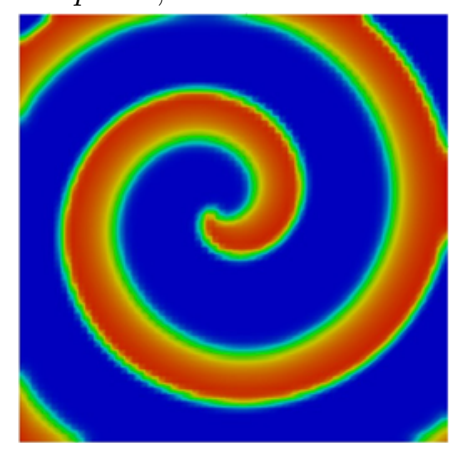

$t=85.0$

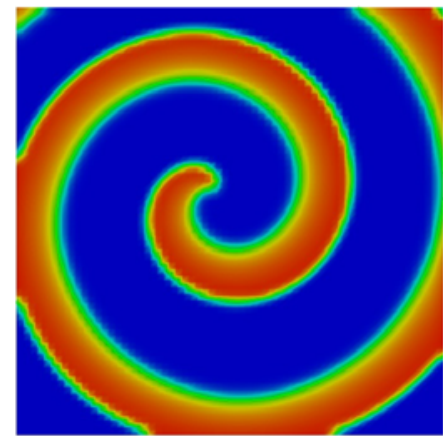

$t=95.0$

Figure 9: Spiral waves of the transmembrane potential induced for the Aliev-Panfilov model at dimensionless times $t=$ 75.0 (left), 85.0 (center), and 95.0 (right) computed with B-spline basis functions of degree $p=1$ and $C^{0}$-continuous built from a mesh of size $h=1 / 32$ with $n_{b f}=4,225$.

cardiac muscle $[16,31,34]$.

We consider for the Aliev-Panfilov model the same data already used in Sect. 3.1 with the computational domain $\Omega=(0,1)^{2}$ and the time step size $\Delta t=0.1$. To set up a test case of re-entrant wave patterns, the initialization is done by applying a stimulus in the wake of a propagating pulse such that the refractory region effectively generates a spiral wave. Numerically speaking, such dynamics requires the approximation of a moving curved front for $v$; thus, we are interested in comparing the simulations of these spiral waves for B-splines of different polynomials degrees $p$ and continuities $C^{0}$ and $C^{p-1}$ of the basis functions. In Fig. 8 we report the numerical results obtained for the IGA spatial approximation with a mesh size $h=1 / 64$ and B-spline basis functions of degree $p=2$ which are globally $C^{1}$-continuous; the results highlight the procedure followed to induce the spiral waves, their formation, and propagation in $\Omega$.

For the example highlighted in Fig. 8 we now compare the numerical results obtained by considering B-spline basis functions of degrees $p=1,2$, and 3 which are globally $C^{0}$ - and $C^{p-1}$-continuous in $\Omega$ over uniform meshes. Specifically, our comparison of the quality of the IGA spatial approximation is based on the number of basis functions $n_{b f}$; the latter is approximately kept constant in the comparison. In this manner, we use meshes of different sizes $h$ to yield about the same $n_{b f}$ for the B-spline bases under consideration ${ }^{1}$

\footnotetext{
${ }^{1}$ We remark that globally $C^{p-1}$-continuous B-spline bases require finer meshes than their $C^{0}$-counterparts to yield the same number of basis functions $n_{b f}$; see [27].
} 

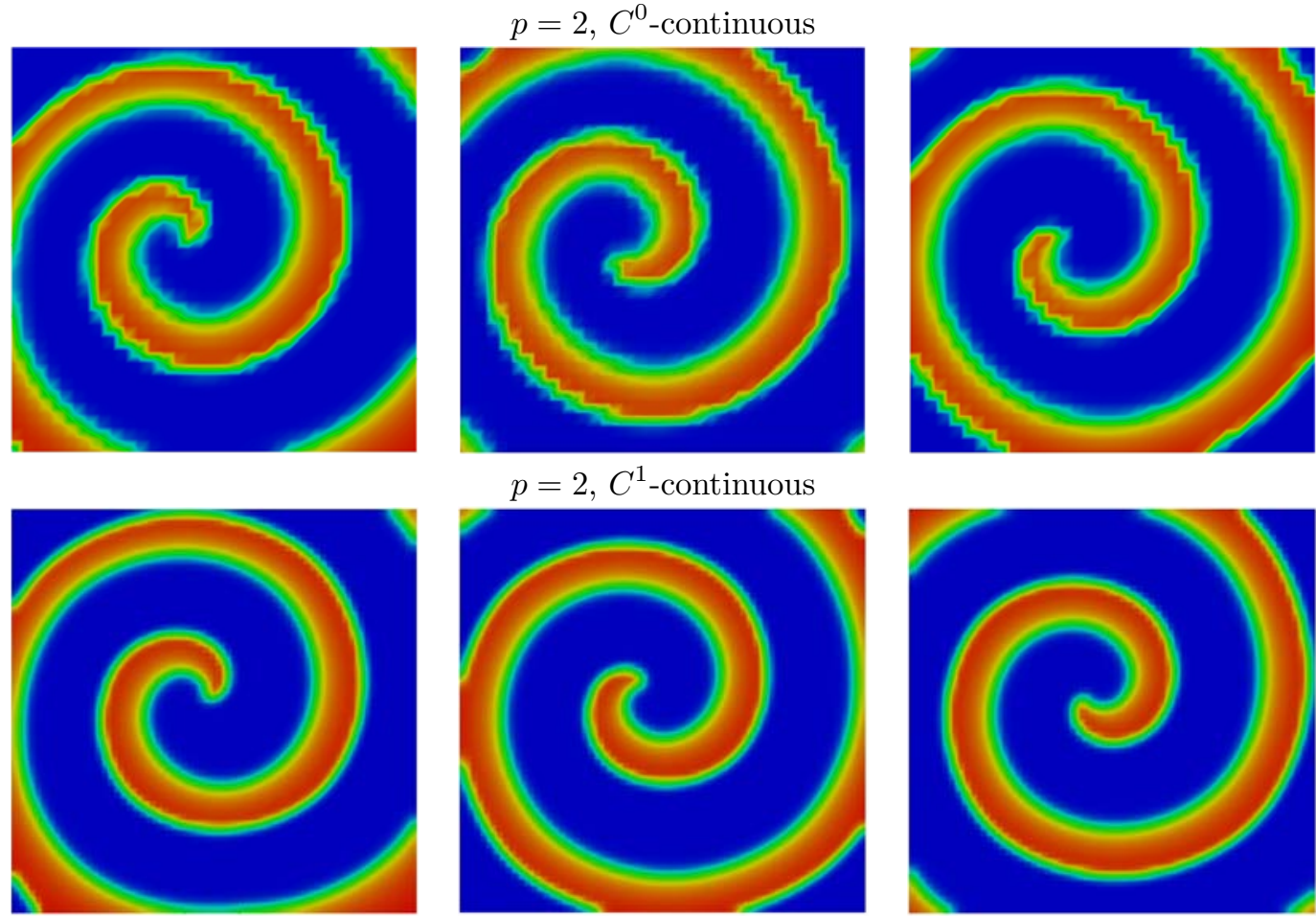

$p=2, C^{1}$-continuous
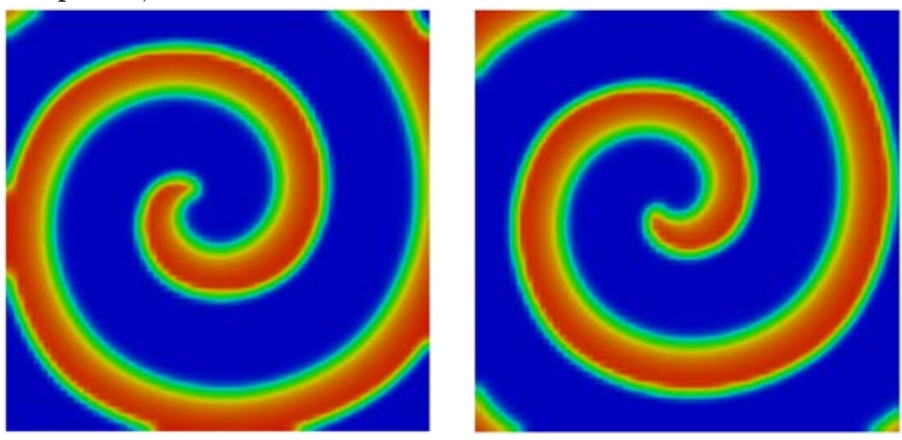

$t=75.0$

$t=85.0$

$t=95.0$

Figure 10: Spiral waves of the transmembrane potential induced for the Aliev-Panfilov model at dimensionless times $t=$ 75.0 (left), 85.0 (center), and 95.0 (right) computed with B-spline basis functions of degree $p=2, C^{0}$-continuous (top) and $C^{1}$ continuous (bottom); $C^{0}$-continuous B-splines are built from a mesh of size $h=1 / 32$ with $n_{b f}=4,225$, while $C^{1}$-continuous B-splines have $h=1 / 64$ with $n_{b f}=4,356$.

are reported in Table 1 where we highlight the corresponding number of mesh elements $n_{e l}$, mesh sizes $h$, and number of basis functions $n_{b f}$. The results obtained at some significant time instances $t$ by means of such bases are highlighted in Figs. 9, 10, and 11 for the degrees $p=1,2$, and 3, respectively. As we can observe, the results obtained for the B-spline bases of degrees $p=1,2$, and 3 and globally $C^{p-1}$-continuous yield comparable results, at least qualitatively, with about the same number of basis functions $n_{b f}$, apart for the tip positions of the spiral waves (partially due to the different induction mechanism used at the discrete level). However, this is not the case for the B-spline bases of degrees $p=2$ and 3 which are only $C^{0}$-continuous; indeed, in these cases, the coarser meshes, used to yield about the same $n_{b f}$, lead to results which exhibit significant grid imprinting. For a more detailed discussion and numerical tests, we refer the interested reader to [49].

In addition, in order to better assess the quality of the IGA spatial approximation, we compare the trajectories of the tips of the spiral waves. Such comparison is made following [16] where the trajectories of fronts' tips are tracked to study the effect on the solutions $v$ of the initial stimuli, specifically the manner in which these are applied and for how long. In [16] several types of tips' trajectories are obtained and analyzed for the 3-variables Fenton-Karma monodomain model [31], even if similar behaviors may can be obtained 


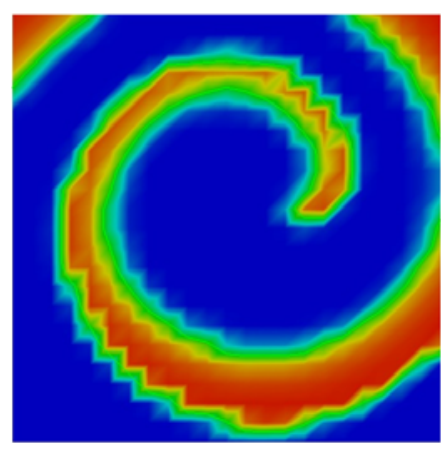

$p=3, C^{0}$-continuous
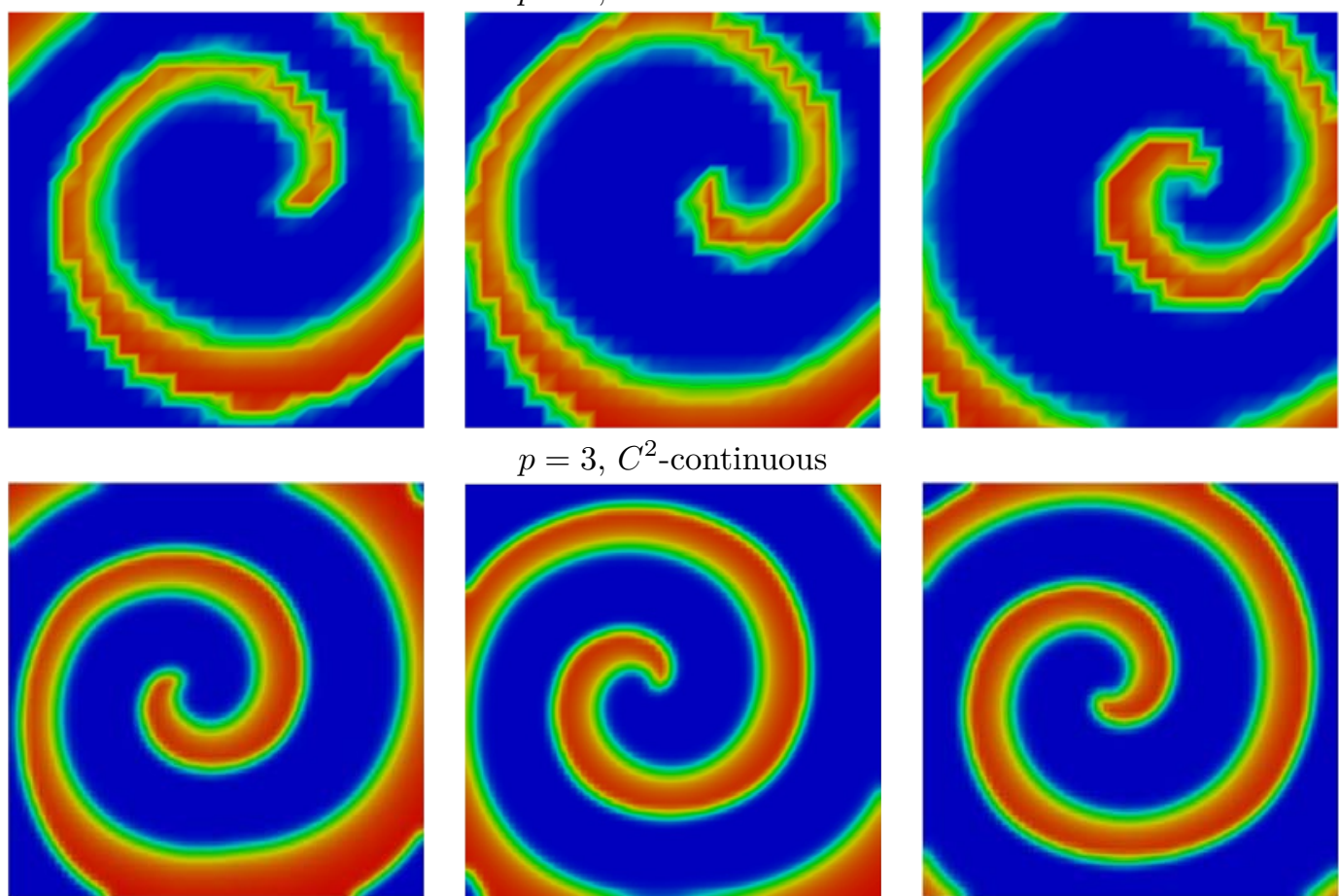

$p=3, C^{2}$-continuous

$t=75.0$

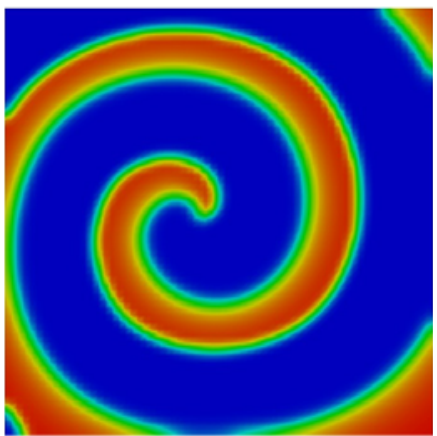

$t=85.0$

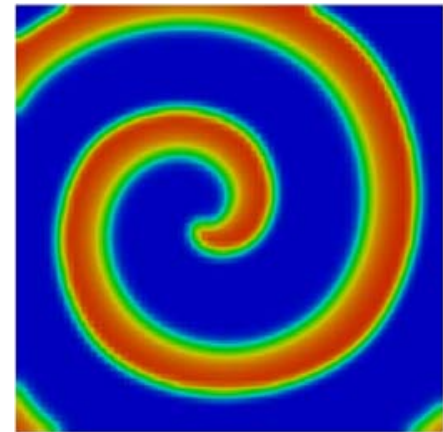

$t=95.0$

Figure 11: Spiral waves of the transmembrane potential induced for the Aliev-Panfilov model at dimensionless times $t=$ 75.0 (left), 85.0 (center), and 95.0 (right) computed with B-spline basis functions of degree $p=3, C^{0}$-continuous (top) and $C^{2}$ continuous (bottom); $C^{0}$-continuous B-splines are built from a mesh of size $h=1 / 22$ with $n_{b f}=4,489$, while $C^{2}$-continuous B-splines from a mesh with $h=1 / 64$ with $n_{b f}=4,489$.

by means of other monodomain models. Here, we extend such analysis to the Aliev-Panfilov model and for the globally $C^{0}$ - and $C^{p-1}$-continuous B-splines of degrees $p=1,2$, and 3 (see also [49]). In Fig. 12 we display the trajectories of the spiral tips corresponding to the spatial discretizations considered in Figs. 9, 10, and 11 for such B-spline bases. The trajectories are determined, over a period of 15.0 dimensionless time units, by tracking the positions of the point laying on the contourline of the transmembrane potential $v=0.5$ which possesses minimum curvature (with sign). The results highlight that the trajectory of the spiral waves' tip should be circular or elliptical and reasonably smooth. Moreover, we observe that the trajectories associated to high order continuous B-spline bases are more accurate and smoother than those associated to bases which are only $C^{0}$-continuous when about the same number of basis functions $n_{b f}$ is involved in the computation. Additional results and comparisons for the $h$ - and $k$-refinements procedures are presented and discussed in [49]; nevertheless, it is quite evident from the tests reported in this paper that the use of the $k$-refinement procedure yields more accurate numerical results. 

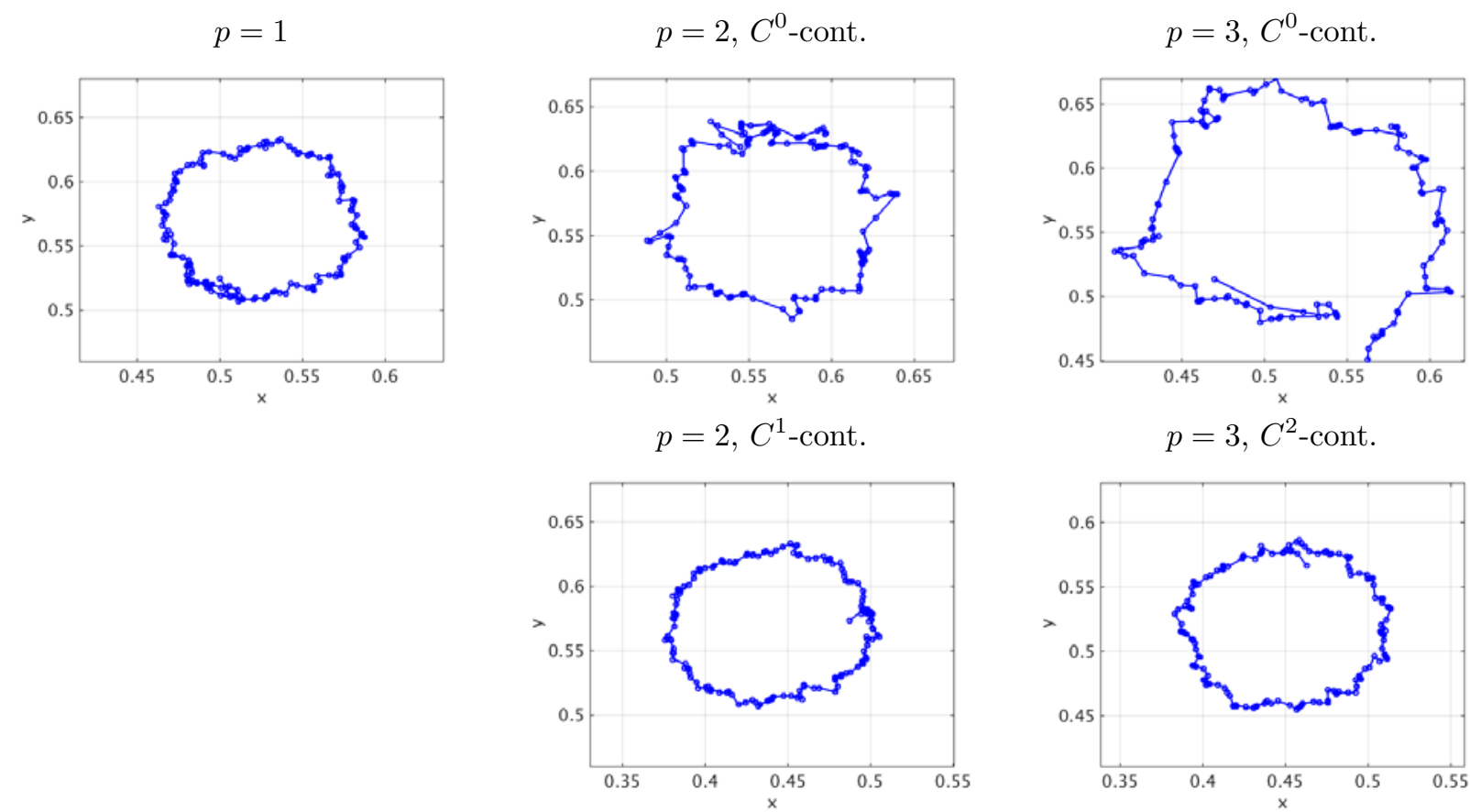

Figure 12: Trajectories of the spiral waves' tips over 15.0 dimensionless time units as computed for the B-spline bases of degrees $p=1$ (left), 2 (center), and 3 (right) and globally $C^{0}$ - (top) and $C^{p-1}$-continuous (bottom); the corresponding transmembrane potentials $v$ are highlighted in Figs. 9, 10, and 11 and the details of the spatial discretizations are reported in Table 1.

\section{Numerical results for a human left atrium}

We solve the Mitchell-Schaeffer monodomain equation [46] presented in Sect. 2.1 by means of IGA on a surface $\Omega \subset \mathbb{R}^{3}$, where $\Omega$, which is represented by means of NURBS, is dimensionally and geometrically similar to the human left atrium (LA). We briefly discuss the procedure followed to represent the human LA by means of NURBS and we discuss our approach for the definition of the anisotropic conductivity of the LA tissue, which is based on the solution of an auxiliary Poisson interpolation problem. Finally, we present the numerical result obtained for the propagation of the transmembrane potential front, with the focus being on the variation of the location of such potential fronts with respect to the number of basis functions used in the IGA spatial approximation.

\subsection{Geometrical representation of the human left atrium by means of NURBS}

We briefly outline the procedure used for the definition of the human LA geometry by means of NURBS. In this respect, the LA can be represented as a surface since the thickness of the atrium wall is small and hence transmural activation differences along the thickness can be assumed to be negligible. The characteristic dimensions of the anatomical features of the human LA are reported in Table 2.

The LA is represented as a mid-surface and built as a single NURBS patch starting from B-spline basis functions of degree $p=2$ along both the parametric directions $\xi_{1}$ and $\xi_{2}$. The generation of the surface 


\begin{tabular}{l|c} 
LA anatomical features & Mean values $[\mathrm{cm}]$ \\
\hline Wall thickness & 0.2 \\
Pulmonary veins diameter (inside) & 1.1 \\
Mitral valve diameter (outside) & 2.9 \\
Anterior-posterior extent & 3.8 \\
Septal-lateral extent & 3.9 \\
LA appendage & \\
$\quad$ Length & 2.9 \\
$\quad$ Diameter (mid) & 1.6
\end{tabular}

Table 2: Characteristic sizes of the anatomical features of the human left atrium (LA). Data taken from [36].
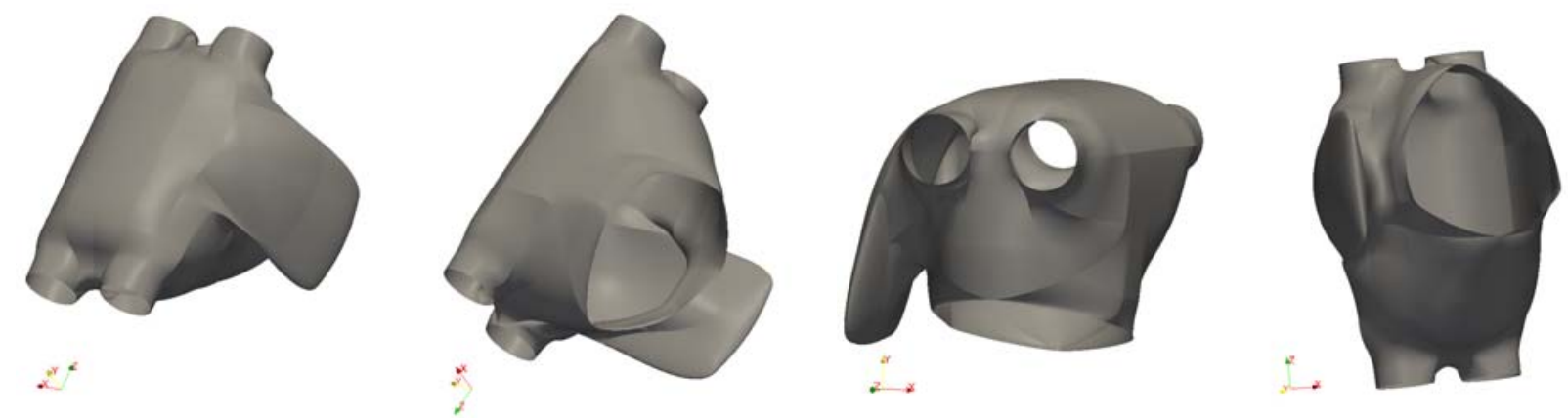

Figure 13: NURBS representation of the LA based on the characteristic sizes of Table 2; views from different angles (the outermost left views are used in Figs. 15 and 16).

\begin{tabular}{c|cccccc} 
Mesh & $\mathcal{T}_{h, 1}$ & $\mathcal{T}_{h, 2}$ & $\mathcal{T}_{h, 3}$ & $\mathcal{T}_{h, 4}$ & $\mathcal{T}_{h, 5}$ & $\mathcal{T}_{h, 6}$ \\
\hline$n_{\text {el }}$ & 2,950 & 10,456 & 22,630 & 39,382 & 60,742 & 86,710 \\
$n_{\text {bf }}$ & 3,100 & 10,772 & 23,052 & 39,940 & 61,436 & 87,540
\end{tabular}

Table 3: Meshes $\mathcal{T}_{h, i}$ used for the numerical simulations of the transmembrane potential on the LA and corresponding number of mesh elements $n_{e l}$ and NURBS basis functions $n_{b f}$ used for the space $\mathcal{N}_{h}$.

starts by considering a cylindrical surface as reference geometrical model, with features added step by step by means of the combined use of the knot insertion procedure ( $h$-refinement, [52]) and extrusion of the newly inserted control points in the physical space; we refer the interested reader to [67] for a more detailed discussion about the generation of NURBS geometries in cardiovascular applications and to [49] regarding the specific LA geometry under consideration. As mentioned, in our geometrical model, we start from the cylindrical shell and we represent the four pulmonary veins by suitable knot insertions along the circumferential direction $\xi_{1}$. Then the oval-shaped opening corresponding to the mitral valve is generated by inserting additional knots in the parametric direction $\xi_{2}$. The resulting representation of the LA by means of NURBS is displayed in Fig. 13 from different points of view. We remark that our geometrical LA model is represented by means of NURBS basis functions of degree $p=2$ and mostly $C^{1}$-continuous along both the parametric directions. Indeed, some "pathological" mesh edges, for which the basis functions are only $C^{0}$-continuous, are maintained in the mesh $\mathcal{T}_{h}$ to facilitate the construction of the NURBS geometry. Our 
basic geometrical representation of the LA involves $n_{b f}=425$ NURBS basis functions and $n_{e l}=345$ mesh elements; we denote the corresponding mesh as $\mathcal{T}_{h, 0}$. The NURBS space $\mathcal{N}_{h}$, which is built using the above mentioned basis, is then successively enriched by means of the $h$-refinement procedure for the use of IGA in the spatial approximation of Eqs. (28)-(30), yet preserving the original representation of $\Omega$ associated to the mesh $\mathcal{T}_{h, 0}$. The number of NURBS basis functions $n_{b f}$ corresponding to the enriched bases and meshes $\mathcal{T}_{h, i}$ are indicated in Table 3.

\subsection{Anisotropic conductivity: the Laplace-Beltrami problem for the definition of the fibers' direction}

The cardiac tissue is strongly anisotropic both in terms of mechanical response and of the conduction velocity on the cells' membrane, as consequence of the local orientation of the cardiomyocytes; such anisotropic behavior affects the conductivity tensor $\mathbf{D}$ in the monodomain equation (5). In this respect, several techniques have been used in literature to reproduce realistic, or at least physically meaningful, anisotropic conductivity tensors for the cardiac tissue. For example, in [63] the authors split the LA into subdomains and then, using characteristic data of the anatomy of the atria as in [37], they defined the conduction tensor piecewise in such subdomain. A similar procedure has been adopted in [21].

In this paper, we built a rule-based fiber-field on the LA surface following the Poisson-based interpolation algorithm of [57] inspired from $[7,66]$ and adapted to the current case for which a surface centerline is not well-defined. The basic idea consists in introducing, at the discrete level, a time-independent potential function $\varphi_{\mathbf{f}, h}$ defined on the LA surface $\Omega$, whose normalized gradient field, say $\mathbf{f}_{h} \in \mathbb{R}^{3}$, yields the fibers' direction as:

$$
\mathbf{f}_{h}=\frac{\nabla_{\Omega} \varphi_{\mathbf{f}, h}}{\left|\nabla_{\Omega} \varphi_{\mathbf{f}, h}\right|} \quad \text { in } \Omega
$$

Specifically, the discrete potential field $\varphi_{\mathbf{f}, h}$, which we use to set the fibers' direction $\mathbf{f}_{h}$ as in (31), is obtained by solving a Laplace-Beltrami problem defined on the surface $\Omega$ with suitable constraints for prescribing a meaningful orientation of the fibers. By assuming $\varphi_{\mathbf{f}, h} \in \mathcal{X}_{h}$, being $\mathcal{X}_{h} \subset C^{0}(\Omega)$ a general finite dimensional function space, we solve the following Laplace-Beltrami problem on $\Omega$ :

$$
\text { find } \varphi_{\mathbf{f}, h} \in \mathcal{Y}_{h} \quad: \quad \int_{\Omega} \nabla_{\Omega} \psi_{h} \cdot \nabla_{\Omega} \varphi_{\mathbf{f}, h} d \Omega=0 \quad \forall \psi_{h} \in \mathcal{Y}_{h}^{0}
$$

with:

$$
\begin{aligned}
& \mathcal{Y}_{h}:=\left\{w_{h} \in \mathcal{X}_{h}: w_{h}\left(\mathbf{x}_{i}\right)=+1 \text { for } i=1,3,4 \text { and } w_{h}\left(\mathbf{x}_{2}\right)=-1\right\}, \\
& \mathcal{Y}_{h}^{0}:=\left\{w_{h} \in \mathcal{X}_{h}: w_{h}\left(\mathbf{x}_{i}\right)=0 \text { for } i=1,2,3,4\right\},
\end{aligned}
$$

where $\mathbf{x}_{1}$ and $\mathbf{x}_{2}$ are two points on $\partial \Omega$ set correspondingly to the portion of the atrium in proximity of the left ventricle, specifically the outermost left and right points as in Fig. 13(right), while the points $\mathbf{x}_{3}$ and $\mathbf{x}_{4}$ lay on $\partial \Omega$ between the pairs of pulmonary veins, respectively. Such constraints are set to prescribe a meaningful orientation of the fibers' field, other than to yield a well-posed Laplace-Beltrami problem (32). 

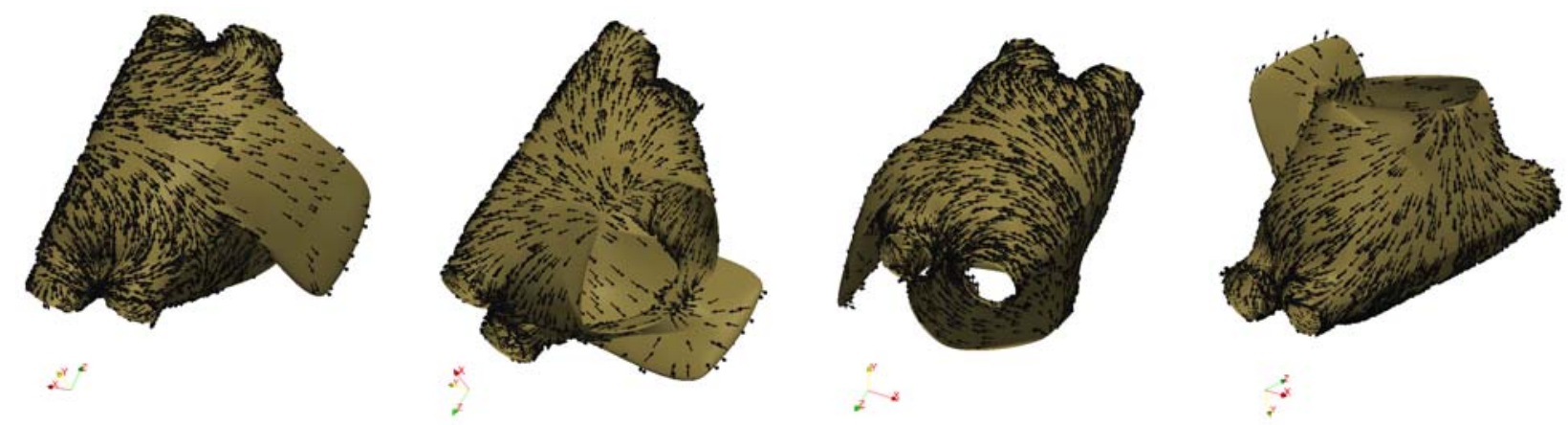

Figure 14: Fibers directions on the LA computed for the mesh $\mathcal{T}_{h, 6}$; views from different angles.

Then, after the fibers' field $\mathbf{f}_{h}$ is computed by solving problem (32) and evaluating the normalized gradient field (31), the discrete anistropic conductivity tensor $\mathbf{D}_{h}$ is defined as:

$$
\mathbf{D}_{h}=D_{i s o}\left(\gamma_{\mathbf{f}} \mathbf{I}+\left(1-\gamma_{\mathbf{f}}\right) \mathbf{F}_{h}\right) \quad \text { in } \Omega,
$$

where $D_{i s o}>0$ is the isotropic conductivity coefficient, $0<\gamma_{\mathbf{f}} \leq 1$ is a parameter setting the level of anisotropy, $\mathbf{I}$ is the identity second order tensor, while $\mathbf{F}_{h}$ is the second order tensor associated to the fibers direction $\mathbf{f}_{h}$, such that $\mathbf{F}_{h, i i}=\mathbf{f}_{h, i}$ for $i=1,2,3$, while $\mathbf{F}_{h, i j}=0$ for $i \neq j$. The rule-based discrete conductivity tensor $\mathbf{D}_{h}$ replaces th tensor $\mathbf{D}$ in the monodomain equation (10) and following ones, thus yielding a transversally isotropic model.

In this work, the Laplace-Beltrami problem (32) is solved by means of NURBS-based IGA, for which we compute the fibers' potential $\varphi_{\mathbf{f}, h}$ by setting $\mathcal{X}_{h} \equiv \mathcal{N}_{h}$ (the NURBS space), and hence the fibers' direction $\mathbf{f}_{h}$ and the anisotropic conductivity tensor $\mathbf{D}_{h}$. We remark that the computation of $\varphi_{\mathbf{f}, h}, \mathbf{f}_{h}$, and $\mathbf{D}_{h}$ depend on the IGA spatial discretization, specifically on the dimension $n_{b f}$ and properties of the NURBS space $\mathcal{N}_{h}$, but not on the geometrical representation of $\Omega$, since we consider meshes $\mathcal{T}_{h, i}$ which preserve the original mapping associated to the mesh $\mathcal{T}_{h, 0}$. We also recall that, in the specific LA geometry under consideration, the NURBS space $\mathcal{N}_{h}$ involves basis functions which are mostly $C^{1}$-continuous in $\Omega$, but also $C^{0}$-continuous along some mesh edges; therefore, the fibers' direction $\mathbf{f}_{h}$ and conductivity tensor $\mathbf{D}_{h}$ may be discontinuous along such mesh edges. Nevertheless, the tensor $\mathbf{D}_{h}$ is evaluated in the monodomain equation (10) only at the Gauss-Legendre quadrature nodes in order to assemble the matrix $\mathbf{K}_{\mathbf{D}}$ in Eq. (24), which are internal to the mesh elements.

In Fig. 14 we show the fibers' direction field $\mathbf{f}_{h}$ computed by means of NURBS-based IGA using the discretization associated to the mesh $\mathcal{T}_{h, 6}$ indicated in Table 3. Such computed fibers field is qualitatively similar to the ones in reported in [21,63]. 

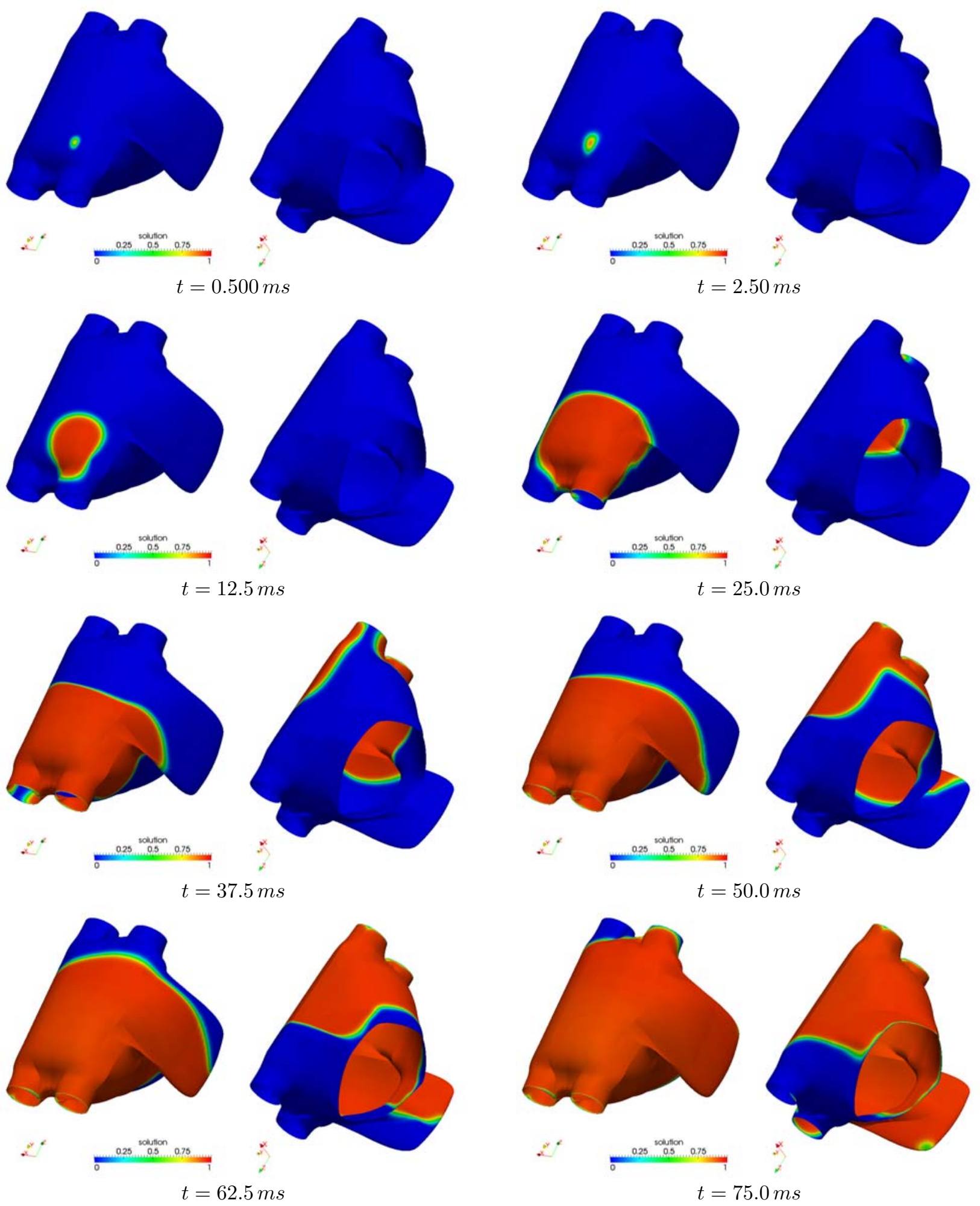

Figure 15: Transmembrane potential (dimensionless) on the LA computed for the mesh $\mathcal{T}_{h, 6}$ at times $0.500 \leq t \leq 75.0 \mathrm{~ms}$; views from different angles. 


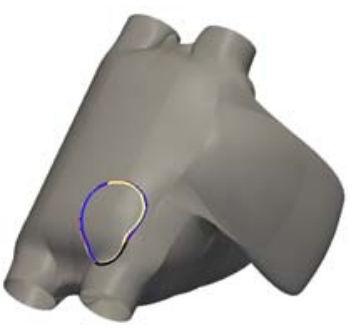

$x^{2}$

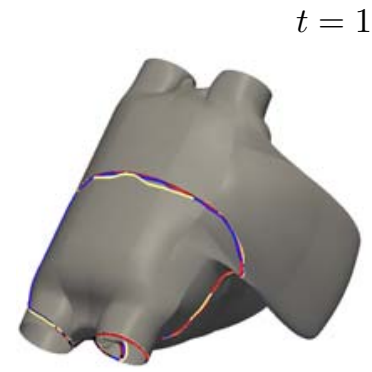

$x^{2}$

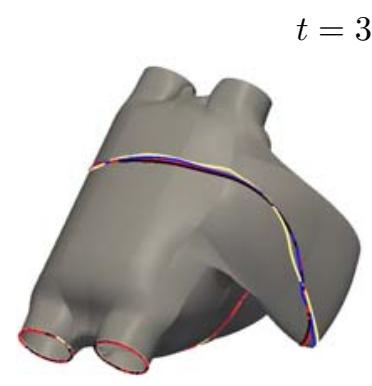

$x^{x}$

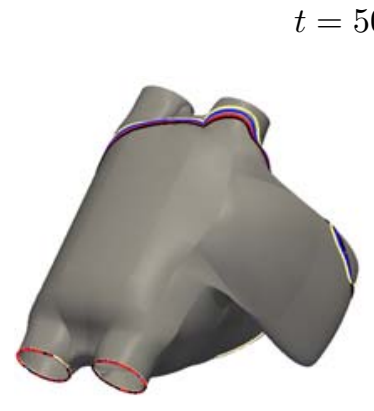

$2^{x}$
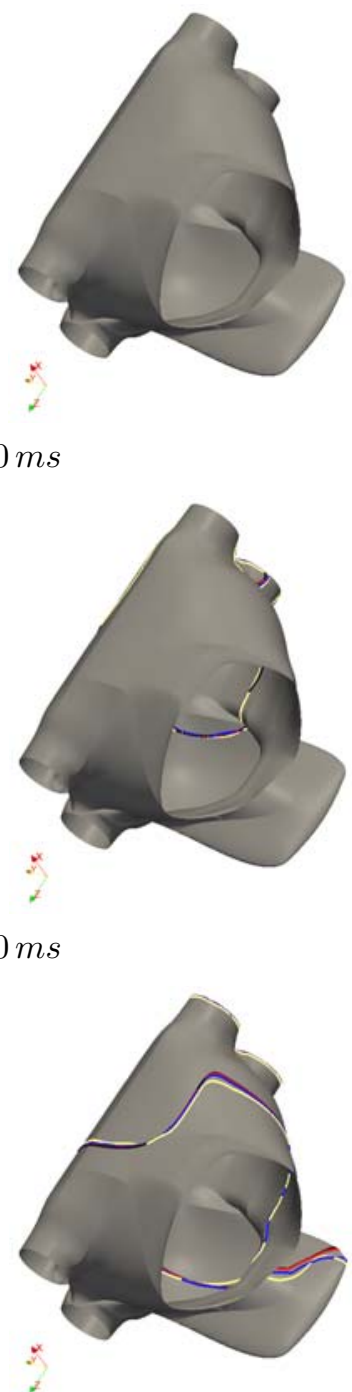

$=50.0 \mathrm{~ms}$

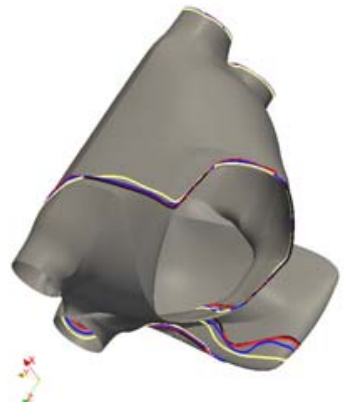

$t=70.0 \mathrm{~ms}$

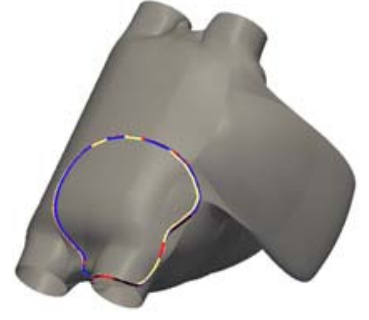

$x^{2}$

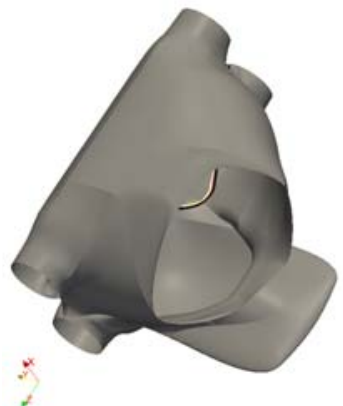

$t=20.0 \mathrm{~ms}$
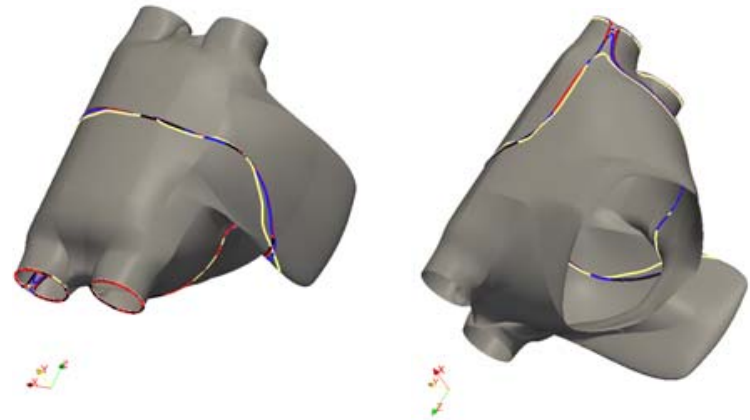

$t=40.0 \mathrm{~ms}$
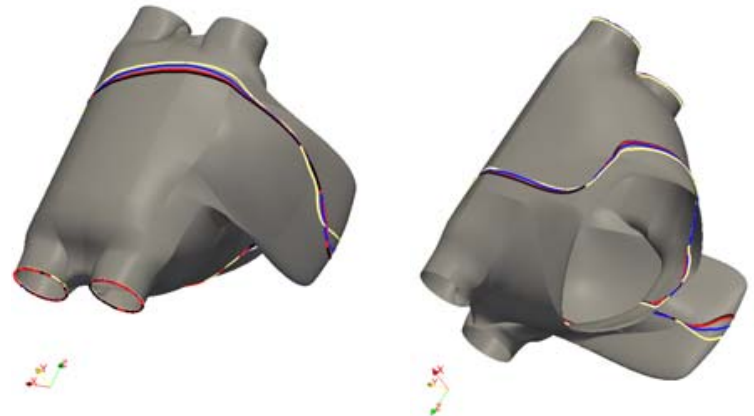

$t=60.0 \mathrm{~ms}$

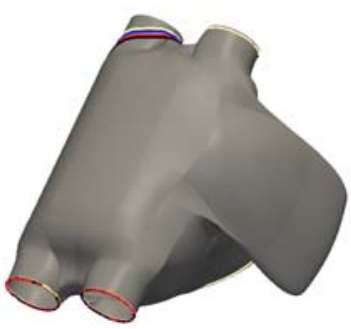

$x^{2}$

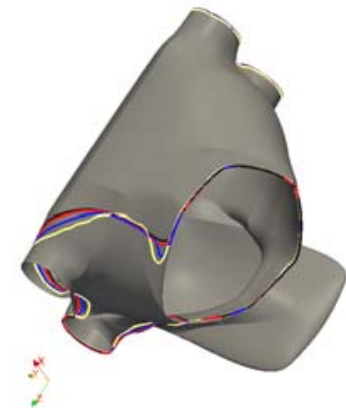

$t=80.0 \mathrm{~ms}$

Figure 16: Comparisons of the transmembrane potential fronts on the LA computed at times $10.0 \leq t \leq 80.0 \mathrm{~ms}$ for the meshes $\mathcal{T}_{h, 1}$ (yellow), $\mathcal{T}_{h, 2}$ (blue), $\mathcal{T}_{h, 4}$ (red), and $\mathcal{T}_{h, 6}$ (black); views from different angles. The front corresponds to the contourline of value 0.5 for the dimensionless transmembrane potential $v$. 


\subsection{Numerical simulation of transmembrane potential on the LA for the Mitchell-Schaeffer model}

We now solve the monodomain problem described by the Mitchell-Schaeffer model by using the numerical scheme described in Eqs. (28)-(30) and based on IGA for the spatial approximation. We use as computational domain $\Omega$ the NURBS representation of the LA highlighted in Fig. 13 and described in Sect. 4.1. The data of the Mitchell-Schaeffer model are the same reported in Sect. 3.1, except for the conductivity tensor $\mathbf{D}$ which is chosen as in Eq. (34) with the isotropic conductivity coefficient $D_{i s o}=0.0100 \frac{\mathrm{cm}^{2}}{\mathrm{~ms}}$ and the anisotropy level $\gamma_{\mathbf{f}}=0.1$. We solve the problem in terms of dimensionless transmembrane potential $v$ and recovery $w$ variables; the value of $v=0$ corresponds to $-70.0 \mathrm{mV}$, while $v=1$ to $30.0 \mathrm{mV}$.

In order to initiate the depolarization of the LA in a physically meaningful manner, we move from the following considerations. The propagation of the electric signal in the LA occurs from four entry points connected to the right atrium (RA), where the depolarization phase originally starts [58]: the Bachmann's bundle, the anterior septum, the posterior septum, and the coronary sinus musculature. In practice, the electric signal can travel from the RA to the LA from one or more of these connection points; the synchronization of the signal entry from these points determine the physiological or pathological propagation of the transmembrane potential on the LA. For our computational model of the LA, in order to avoid synchronization issues, we assume that the initialization of the depolarization occurs only at the Bachmann's bundle, which is approximately located in the interior LA wall close to the right superior pulmonary vein. More specifically, in our model, we initialize the depolarization of the LA by forcing the dimensionless transmembrane potential and recovery variables to be equal to 1.0 in a region of diameter $0.2 \mathrm{~cm}$ corresponding to the Bachmann's bundle and for a time inferior to $T_{s}=0.0400 \mathrm{~ms}$; the initial value of the dimensionless transmebrane and recovery variables is set equal to 0.0 .

We solve the monodomain equation up to the final time $T=400 \mathrm{~ms}$ by prescribing a homogeneous Dirichlet boundary condition for the potential $v$ at the boundary $\partial \Omega$ of the LA; for the time discretization we consider the time step size $\Delta t=0.0200 \mathrm{~ms}$. We report in Fig. 15 the evolution of the dimensionless transmembrane potential on the LA using the spatial discretization corresponding to the mesh $\mathcal{T}_{h, 6}$ of Table 3. The evolution of the transmembrane potential covers the depolarization phase; the region where the initialization of the depolarization occurs is visualized in Fig. 15(top-left).

We remark that we use NURBS basis functions of degree $p=2$ and mostly $C^{1}$-continuous, except at some mesh edges where these are $C^{0}$-continuous. Therefore, as already discussed in Sect. 3.1 for the propagation of the front velocity for the Mitchell-Schaeffer model, we can reasonably expect that such velocity is accurately represented already for relatively coarse meshes and with a "small" number of basis functions $n_{b f}$. To confirm this, we solve the monodomain problem on the LA by means of IGA with the spatial discretizations indicated in Table 3. We remark that, for all the meshes $\mathcal{T}_{h, 2}-\mathcal{T}_{h, 6}$, we obtain results which are qualitatively and quantitatively very similar to the ones of Fig. 15. We highlight this aspect by comparing in Fig. 16 the front locations of the dimensionless transmebrane potential computed for the 

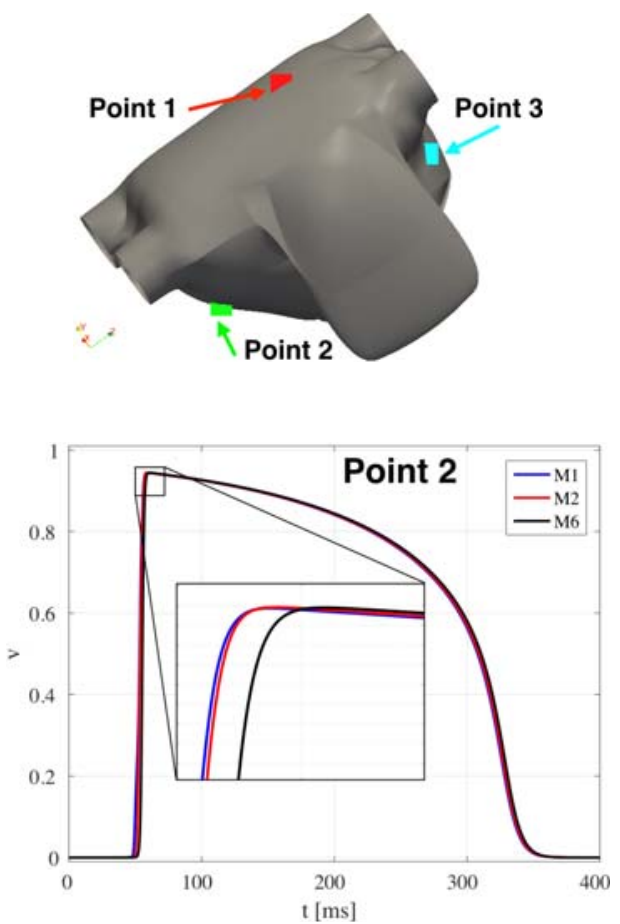
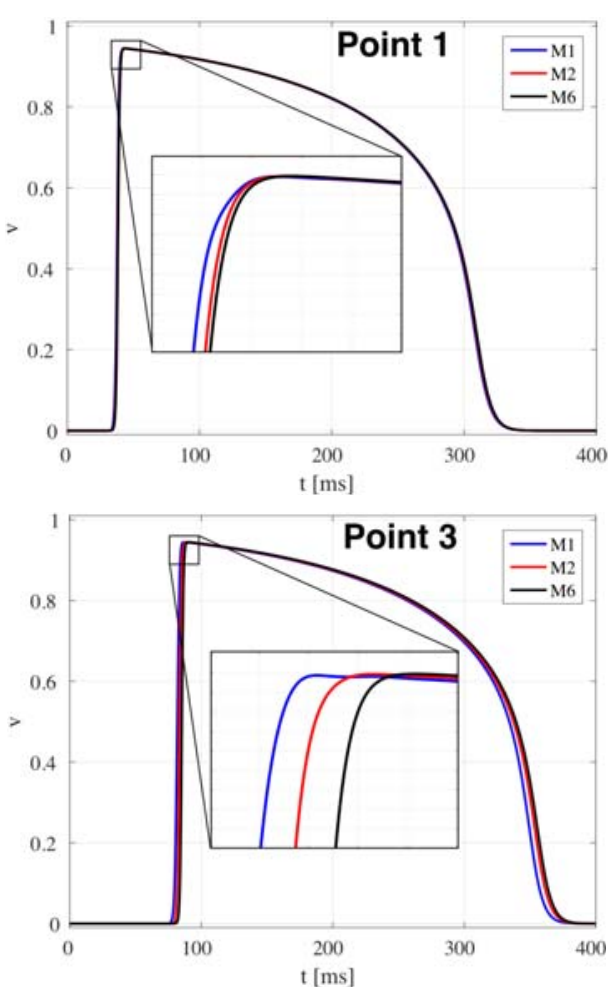

Figure 17: Action potential at three points on the LA surface computed for the meshes $\mathcal{T}_{h, 1}$ (blue), $\mathcal{T}_{h, 2}\left(\right.$ red), and $\mathcal{T}_{h, 6}($ black).

discretizations associated to the meshes $\mathcal{T}_{h, 1}, \mathcal{T}_{h, 2}, \mathcal{T}_{h, 4}$, and $\mathcal{T}_{h, 6}$. The results associated to the meshes $\mathcal{T}_{h, 5}$ and $\mathcal{T}_{h, 6}$ are in practice coincident also for "large" values of the time $t$. Therefore, we remark that accurate results can be obtained with relatively coarse discretizations if smooth NURBS basis functions are used for the IGA spatial approximation. This is also confirmed in Fig. 17 by comparing the action potentials computed for the meshes $\mathcal{T}_{h, 1}, \mathcal{T}_{h, 2}$, and $\mathcal{T}_{h, 6}$ in three points on the LA.

\section{Conclusions}

In this paper we considered IGA for the spatial approximation of cardiac electrophysiology models, specifically based on the monodomain equation coupled to the Mitchell-Schaeffer [46] and Aliev-Panfilov [3] models. We qualitatively and quantitatively addressed and discussed the numerical results for benchmark test problems with the focus being on the properties on the basis functions, namely B-splines and NURBS, used in the IGA approximation of the monodomain equation. Indeed, other than the geometrical advantages allowed by the use of the isogeometric concept in the representation of the computational domain, the approximation properties of IGA also depend on the specific basis functions used. In this respect, it is well know in literature $[2,24,25,27,30,39,40,62]$ that globally, high order continuous basis functions as B-splines and NURBS yield very accurate and computationally efficient approximations of several classes of PDEs, including problems exhibiting smooth but sharp layer and interfaces [33, 45], possibly over surfaces 
$[6,28]$. In this paper we showed that, also for the cardiac elecrophysiology models under consideration, Bspline basis functions of degree $p \geq 2$ and globally $C^{p-1}$-continuous over the computational domain provide more accurate results than their $C^{0}$-counterpart when about the same number of basis functions is used in the spatial discretization. Specifically, the results obtained for traveling fronts of the transmembrane potential $v$, both for straight fronts or when spiral waves are developed, indicate that B-splines of degrees $p=2$ and 3 which are $C^{1}$ - and $C^{2}$-continuous, respectively, require a relatively small number of degrees of freedom for the spatial approximation. Thus, in the case such bases are used or the $k$-refinement procedure is adopted for their enrichment, the numerical solution of the monodomain problems may be more accurate and eventually more efficient than with $C^{0}$-continuous basis functions, like those used with the standard Finite Element method.

In addition, we approximated and solved by means of IGA the monodomain equation based on the Mitchell-Schaeffer model on a realistic geometry of the human left atrium (LA), which we geometrically represented as a NURBS surface. In particular, we highlighted that the spatial approximation based on NURBS basis functions of degree $p=2$ and mostly $C^{1}$-continuous over the LA geometry yields very accurate results already for relatively coarse meshes and few degrees of freedom, thus resulting potentially advantageous with respect to approximations based on the Finite Element method.

\section{Acknowledgments}

A. Bartezzaghi, L. Dedè, and A. Quarteroni acknowledge the financial support of the Swiss National Science Foundation through the project "Isogeometric Analysis for Partial Differential Equations: surface models and optimization problems in Haemodynamics" (project $\sharp 147033,2014-2016$ ).

\section{References}

[1] R.A. Adams. Sobolev Spaces. Academic Press, New York, 1975.

[2] I. Akkerman, Y. Bazilevs, V.M. Calo, T.J.R. Hughes, and S. Hulshoff. The role of continuity in residual-based variational multiscale modeling of turbulence. Comput. Mech., 41(3):371-378, 2008.

[3] R.R. Aliev and A.V. Panfilov. A simple two-variable model of cardiac excitation. Chaos Soliton. Fract., 7(3):293-301, 1996.

[4] F. Auricchio, F. Calabrò, T.J.R. Hughes, A. Reali, and G. Sangalli. A simple algorithm for obtaining nearly optimal quadrature rules for NURBS-based Isogeometric Analysis. Comput. Meth. Appl. Mech. Eng., 249-252:15-27, 2012.

[5] F. Auricchio, L. Beirão da Veiga, T.J.R. Hughes, A. Reali, and G. Sangalli. Isogeometric collocation methods. Math. Models Meth. Appl. Sci., 20(11):2075-2107, 2010.

[6] A. Bartezzaghi, L. Dedè, and A. Quarteroni. Isogeometric Analysis for high order Partial Differential Equations on surfaces. Comput. Meth. Appl. Mech. Eng., 295:446-469, 2015.

[7] J.D. Bayer, R.C. Blake, G. Plank, and N.A. Trayanova. A novel rule-based algorithm for assigning myocardial fiber orientation to computational heart models. Ann. Biomed. Eng., 40(10):54, 2012.

[8] Y. Bazilevs, L. Beirão da Veiga, J.A. Cottrell, T.J.R. Hughes, and G. Sangalli. Isogeometric Analysis: approximation, stability, and error estimates for $h$-refined meshes. Math. Models Meth. Appl. Sci., 16(7):1031-1090, 2006.

[9] M. Bendahmane, R. Bürger, and R. Ruiz-Baier. A multiresolution space-time adaptive scheme for the bidomain model in electrocardiology. Numer. Methods Partial Differential Equations, 26(6):1377-1404, 2010.

[10] M. Berger. A Panoramic View of Riemannian Geometry. Springer-Verlag, Berlin and Heidelberg, 2003.

[11] Y. Bourgault, Y. Coudière, and C. Pierre. Existence and uniqueness of the solution for the bidomain model used in cardiac electrophysiology. Nonlinear Anal.-Theor., 10(1):458-482, 2009.

[12] C.D. Cantwell, S. Yakovlev, R.M. Kirby, N.S. Peters, and S.J. Sherwin. High-order spectral/hp element discretisation for reaction-diffusion problems on surfaces: Application to cardiac electrophysiology. J. Comput. Phys., 257:813-829, 2014. 
[13] D. Chapelle, A. Collin, and J.-F. Gerbeau. A surface-based electrophysiology model relying on asymptotic analysis and motivated by cardiac atria modeling. Math. Mod. Meth. Appl. S., 23(14):2749-2776, 2013.

[14] L.A. Charawi. Isogeometric overlapping Schwarz preconditioners in computational electrocardiology. PhD thesis, Univeristà degli Studi di Milano, 2014.

[15] L.A. Charawi. Isogeometric overlapping additive Schwarz solvers for the bidomain system. In Th. Dickopf, M.J. Gander, L. Halpern, R. Krause, and L.F. Pavarino, editors, Domain Decomposition Methods in Science and Engineering XXII, Lecture Notes in Computational Science and Engineering. Springer, 2015 (to appear).

[16] E.M. Cherry and F. Fenton. Visualization of spiral and scroll waves in simulated and experimental cardiac tissue. New J. Phys, 10:1-43, 2008 .

[17] J. Chung and G.M. Hulbert. A time integration algorithm for structural dynamics with improved numerical dissipation: the generalized $\alpha$-method. J. Appl. Mech., 60(2):371-375, 1993.

[18] P. Colli Franzone, P. Deuflhard, B. Erdmann, J. Lang, and L.F. Pavarino. Adaptivity in space and time for reactiondiffusion systems in electrocardiology. SIAM J. Sci. Comput., 28(3):942-962, 2006.

[19] P. Colli Franzone, L.F. Pavarino, and S. Scacchi. Mathematical and numerical methods for reaction-diffusion models in electrocardiology. In Modeling of Physiological Flows, pages 107-141. Springer, 2012.

[20] P. Colli Franzone, L.F. Pavarino, and S. Scacchi. Mathematical Cardiac Electrophysiology. Modeling, Simulation and Applications. Springer, 2014.

[21] A. Collin, J.F. Gerbeau, M. Hocini, M. Haïssaguerre, and D. Chapelle. Surface-based electrophysiology modeling and assessment of physiological simulations in atria. FIMH - 7th Int. Conf. on Funct. Imag. and Mod. of the Heart, 7945:352$359,2013$.

[22] M.A. Colman, S.J. Castro, E.A. Perez Alday, J.C. Hancox, C. Garratt, and H. Zhang. Recent progress in multi-scale models of the human atria. Drug Discov. Today Dis. Models, 2014. DOI: 10.1016/j.ddmod.2014.04.003.

[23] J.A. Cottrell, T.J.R. Hughes, and Y. Bazilevs. Isogeometric Analysis. Toward Integration of CAD and FEA. Wiley, 2009.

[24] J.A. Cottrell, T.J.R. Hughes, and A. Reali. Studies of refinement and continuity in Isogeometric structural analysis. Comput. Meth. Appl. Mech. Eng., 196(41-44):4160-4183, 2007.

[25] J.A. Cottrell, A. Reali, Y. Bazilevs, and T.J.R. Hughes. Isogeometric analysis of structural vibrations. Comput. Methods Appli. Mech. Eng., 195(41-43):5257-5296, 2006.

[26] M. Courtemanche, R.J. Ramirez, and S. Nattel. Ionic mechanisms underlying human atrial action potential properties: insights from a mathematical model. American J. Phys. - Heart Circul. Phys., 275(1):H301-H321, 1998.

[27] L. Dedè, C. Jäggli, and A. Quarteroni. Isogeometric numerical dispersion analysis for two-dimensional elastic wave propagation. Comput. Meth. Appl. Mech. Eng., 284(320-348), 2015.

[28] L. Dedè and A. Quarteroni. Isogeometric Analysis for second order Partial Differential Equations on surfaces. Comput. Meth. Appl. Mech. Eng., 284:807-834, 2015.

[29] M.C. Delfour and J.-P. Zolésio. Shapes and Geometries: Analysis, Differential Calculus, and Optimization. SIAM, Philadelphia, PA, USA, 2001.

[30] J.A. Evans, Y. Bazilevs, I. Babuška, and T.J.R. Hughes. $n$-widths, sup-infs, and optimality ratios for the $k$-version of the Isogeometric finite element method. Comput. Meth. Appl. Mech. Eng., 198(21-26):1726-1741, 2009.

[31] F. Fenton and A. Karma. Vortex dynamic in three-dimensional continuous myocardium with fiber rotation: Filament instability and fibrillation. Chaos, 8:20-47, 1998.

[32] F.H. Fenton and E. M. Cherry. Models of cardiac cell. 3(8):1868, 2008. revision $\sharp 91508$.

[33] H. Gómez, V.M. Calo, Y. Bazilevs, and T.J.R. Hughes. Isogeometric analysis of the Cahn-Hilliard phase-field model. Comput. Methods Appli. Mech. Eng., 197(49-50):4333-4352, 2008.

[34] R. Gray and J. Jalife. Spiral waves and the heart. Int. J. Bifurcation Chaos App. Sci. Eng., 6:415, 1996.

[35] A.C. Guyton and J.E. Hall. Medical Physiology. Saunders, 1961.

[36] D.M. Harrild and C.S. Henriquez. A computer model of normal conduction in the human atria. Cir. Res., 87:e25-e36, 2000.

[37] S.Y. Ho, R.H. Anderson, and D. Sánchez-Quintana. Atrial structure and fibers: morphologic bases of atrial conduction. Card. R., 54:325-336, 2002.

[38] T.J.R. Hughes, J.A. Cottrell, and Y. Bazilevs. Isogeometric Analysis: CAD, finite elements, NURBS, exact geometry and mesh refinement. Comput. Meth. Appl. Mech. Eng., 194(39-41):4135-4195, 2005.

[39] T.J.R. Hughes, J.A. Evans, and A. Reali. Finite element and NURBS approximations of eigenvalue, boundary-value, and initial-value problems. Comput. Meth. Appl. Mech. Eng., 272:290-320, 2014.

[40] T.J.R. Hughes, A. Reali, and G. Sangalli. Duality and unified analysis of discrete approximations in structural dynamics and wave propagation: comparison of p-method finite elements with k-method nurbs. Comput. Meth. Appl. Mech. Eng., 194(49-50):4104-4124, 2008.

[41] A. Hundsdorfer and J.G. Verwer. Numerical Solution of Time-Dependent Advection-Diffusion-Reaction Equations. Spinger, Amsterdam, 2003.

[42] J.P. Keener and J. Sneyd. Mathematical Physiology, volume 8. Springer, 1998.

[43] S. Krishnamoorthi, M. Sarkar, and W.S. Klug. Numerical quadrature and operator splitting in finite element methods for cardiac electrophysiology. Int. J. Num. Meth. Biomed. Engng., 29(11):1243-1266, 2013.

[44] K. Kunisch and A. Marica. Well-posedness for the Mitchell-Schaeffer model of the cardiac membrane. SFB-Report No. 2013-018, 2013.

[45] J. Liu, L. Dedè, J.A. Evans, M.J. Borden, and T.J.R. Hughes. Isogeometric Analysis of the advective Cahn-Hilliard equation: spinodal decomposition under shear flow. J. Comp. Phys., 242:321-350, 2013.

[46] C.C. Mitchell and D.G. Schaeffer. A two-current model for the dynamics of cardiac membrane. Bull. Math. Biol., 
65(5):767-793, 2003.

[47] S.A. Niederer, E. Kerfoot, A.P. Benson, M.O. Bernabeu, O. Bernus, C. Bradley, E.M. Cherry, R. Clayton, F.H. Fenton, and A. et al. Garny. Verification of cardiac tissue electrophysiology simulators using an n-version benchmark. Phil. Trans. R. Soc. A, 369(1954):4331-4351, 2011.

[48] A. Nygren, C. Fiset, L. Firek, J. W. Clark, D. S. Lindblad, R. B. Clark, and W. R. Giles. Mathematical model of an adult human atrial cell. Circul. Research, 82(1):63-81, 1998.

[49] A. Patelli. Isogeometric analysis of electrophysiological models on surfaces. Master's thesis, École Polytechnique Fédérale de Lausanne, 2014.

[50] P. Pathmanathan, M.O. Bernabeu, S.A. Niederer, D.J. Gavaghan, and D. Kay. Computational modelling of cardiac electrophysiology: explanation of the variability of results from different numerical solvers. Int. J. Numer. Methods Biomed. Engr., 28(8):890-903, 2012.

[51] M. Pennacchio, G. Savarè, and P. Colli Franzone. Multiscale modeling for the bioelectric activity of the heart. SIAM J. Math. Anal., 37(4):1333-1370, 2005.

[52] L. Piegl and W. Tiller. The NURBS book. Springer-Verlag, New York, 1997.

[53] G. Plank, L. Zhou, J.L. Greenstein, S. Cortassa, R.L. Winslow, B. O'Rourke, and N.A. Trayanova. From mitochondrial ion channels to arrhythmias in the heart: computational techniques to bridge the spatio-temporal scales. Phil. Trans. R. Soc. A, 366(1879):3381-3409, 2008.

[54] A. Quarteroni. Numerical Models for Differential Problems, volume 8 of Modeling, Simulation and Applications. SpringerVerlag, 2014.

[55] A. Quarteroni, T. Lassila, S. Rossi, and R. Ruiz-Baier. Integrated heart - coupling multiscale and multiphysics models for the simulation of the cardiac function. Comput. Methods Appl. Mech. Eng.

[56] A. Quarteroni, R. Sacco, and F. Saleri. Numerical Mathematics. Springer-Verlag, Berlin and Heidelberg, 2007.

[57] S. Rossi, T. Lassila, R. Ruiz-Baier, A. Sequeira, and A. Quarteroni. Thermodynamically consistent orthotropic activation model capturing ventricular systolic wall thickening in cardiac electromechanics. Eur. J. Mech. - A/Solids, 48(1):129-142, 2014.

[58] S. Sakamoto, T. Nitta, Y. Ishii, Y. Miyagi, H. Ohmori, and K. Shimizu. Interatrial electrical connections: the precise location and preferental conduction. J. Card. Electro., 16:1077-1086, 2005.

[59] D. Schillinger, J.A. Evans, A. Reali, M.A. Scott, and T.J.R. Hughes. Isogeometric collocation: cost comparison with Galerkin methods and extension to adaptive hierarchical NURBS discretizations. Comput. Meth. Appl. Mech. Eng., 267:170-232, 2013.

[60] N. Smith, D. Nickerson, E. Crampin, and P. Hunter. Multiscale computational modelling of the heart. Acta Num., 13(1):371-431, 2004

[61] J. Southern, G.J. Gorman, M.D. Piggott, and P.E. Farrell. Parallel anisotropic mesh adaptivity with dynamic load balancing for cardiac electrophysiology. J. Comput. Sci., 3(1):8-16, 2012.

[62] A. Tagliabue, L. Dedè, and A. Quarteroni. Isogeometric Analysis and error estimates for high order partial differential equations in fluid dynamics. Comput. ES Fluids, 102:277-303, 2014.

[63] C. Tobón, C.A. Ruiz-Villa, E. Heidenreich, L. Romero, and F. Hornero. A three-dmensional human atrial model with fiber orientation. electrograms and arrhythmic activation patterns relationship. PLoS ONE, 8:1-13, 2013.

[64] E. Vigmond, F. Vadakkumpadan, V. Gurev, H. Arevalo, M. Deo, G. Plank, and N. Trayanova. Towards predictive modelling of the electrophysiology of the heart. Exp. Physiol., 94(5):563-577, 2009.

[65] K. Wang, S.Y. Ho, D.G. Gibson, and R.H. Anderson. Architecture of atrial musculature in humans. Brit. Heart J., 73(6):559-565, 1995.

[66] J. Wong and E. Kuhl. Generating fibre orientation maps in human heart models using poisson interpolation. Comput. Meth. Biomech. Biomed. Engrg., 17(11):1217-1226, 2014.

67] Y. Zhang, Y. Bazilevs, S. Goswami, C. Bajaj, and T.J.R. Hughes. Patient-specific vascular NURBS modeling for Isogeometric Analysis of blood flow. Comp. Meth. Appl. Mech. Eng., 196(29-30):2943-2959, 2007. 